\title{
Hopf Algebroid Symmetry of Abstract Frobenius Extensions of Depth 2
}

\author{
Gabriella Böhm ${ }^{1}$ And Kornél Szlachányi ${ }^{2}$
}

\begin{abstract}
We study Frobenius 1-morphisms $\iota$ in an additive bicategory $\mathcal{C}$ satisfying the depth 2 condition. We show that the 2-endomorphism rings $\mathcal{C}^{2}(\iota \times \bar{\iota}, \iota \times \bar{\iota})$ and $\mathcal{C}^{2}(\bar{\iota} \times \iota, \bar{\iota} \times \iota)$ can be equipped with dual Hopf algebroid structures. We prove also that a Hopf algebroid appears as the solution of the above abstract symmetry problem if and only if it possesses a two sided non-degenerate integral.
\end{abstract}

\section{Introduction}

In the classification of extensions of von Neumann algebras 'quantum groups' and 'quantum groupoids' play important role.

The starting point was the result of [27, 12] proving that an irreducible finite index depth 2 (or D2 for short) extension of von Neumann factors can be realized as a crossed product with a finite dimensional $C^{*}$-Hopf algebra - that is the symmetry of the extension is described by a finite dimensional $C^{*}$-Hopf algebra. About related results see also [20, 7, 8, 30, 19]. The D2 property means that the derived tower of relative commutants $N^{\prime} \cap M \subset N^{\prime} \cap M_{1} \subset N^{\prime} \cap M_{2} \subset \ldots$ of the Jones tower $N \subset M \subset M_{1} \subset M_{2} \ldots$ is also a Jones tower 10 .

Allowing for reducible finite index D2 extensions of $I I_{1}$ von Neumann factors in 15 and of von Neumann algebras with finite centers in [18] the symmetry of the extension was shown to be described by a finite dimensional $C^{*}$-weak Hopf algebra introduced in [4, 17, 3]. A Galois correspondence has been established in [16] in the case of finite index finite depth extensions of $I I_{1}$ factors. The infinite index D2 case has been treated in [9] for arbitrary von Neumann algebras endowed with a regular operator valued weight.

\footnotetext{
${ }^{1}$ Research Institute for Particle and Nuclear Physics, Budapest, H-1525 Budapest 114, P.O.B. 49, Hungary E-mail: BGABR@rmki.kfki.hu

Supported by the Hungarian Scientific Research Fund, OTKA - T 034 512, FKFP - 0043/2001 and the Bolyai János Fellowship

${ }^{2}$ Research Institute for Particle and Nuclear Physics, Budapest, H-1525 Budapest 114, P.O.B. 49, Hungary E-mail: SZLACH@rmki.kfki.hu

Supported by the Hungarian Scientific Research Fund, OTKA - T 034512.
} 
In [1] the analysis was extended to extensions of rings $N \rightarrow M$. The D2 condition has been generalized as

i) the $N$-M bimodule ${ }_{N} M_{N} \underset{N}{\otimes}{ }_{N} M_{M}$ is a direct summand in a finite direct sum of copies of ${ }_{N} M_{M}$,

ii) the $M-N$ bimodule ${ }_{M} M_{N} \underset{N}{\otimes}{ }_{N} M_{N}$ is a direct summand in a finite direct sum of copies of ${ }_{M} M_{N}$. A D2 extension of von Neumann algebras is D2 in this sense.

The symmetry of a D2 ring extension $N \rightarrow M$ was shown in [1] to be described by a finite bialgebroid. That is the step 2 centralizer rings $A:=\operatorname{End}_{N} M_{N}$ - the endomorphism ring of the bimodule ${ }_{N} M_{N}-$ and $B:=(M \underset{N}{\otimes} M)^{N}$ - the center the bimodule ${ }_{N} M_{N} \underset{N}{\otimes}{ }_{N} M_{N}$ with multiplication $\left(b_{1} \otimes b_{2}\right)\left(b_{1}^{\prime} \otimes b_{2}^{\prime}\right):=$ $b_{1}^{\prime} b_{1} \otimes b_{2} b_{2}^{\prime}$ - carry dual bialgebroid structures over the basis $C_{N}(M)$ - the centralizer of $N$ in $M$. The bialgebroid $A$ has a natural left action on $M$. Under the additional assumption that the module $M_{N}$ is balanced, the $A$-invariant subalgebra of $M$ was shown to be $N$. Also $B$ acts naturally on $\operatorname{End}_{N} M$ with the invariant subalgebra isomorphic to $M$.

The bialgebroid - or Takeuchi $\times_{L^{-}}$bialgebra - is a generalization of the bialgebra in the sense that instead of being an $L$-algebra it is a bimodule over a non-commutative base ring $L$. The bialgebroid definitions [28, 13, 29, 21, 25] were shown to be equivalent in [5]. The bialgebroid $A$ over the base $L$ is finite if the $L$-module structures on $A$ are finitely generated projective. By the results in [11] in this case the $L$-duals of $A$ also carry bialgebroid structures. We summarize the basic definitions of the theory of bialgebroids in Section 2 ,

In the Section 3 of [2] the D2 ring extension $N \rightarrow M$ was supposed to be also Frobenius. This means the existence of a Frobenius map $\Phi:{ }_{N} M_{N} \rightarrow N$ possessing a quasi-basis $x_{j}, y_{j} \in M$ satisfying $\sum_{j} y_{j} \Phi\left(x_{j} m\right)=m=\sum_{j} \Phi\left(m y_{j}\right) x_{j}$ for all $m \in M$. Under this assumption the above rings $A$ and $B$ carry more structure then just being bialgebroids. Namely, fixing a Frobenius map $M \rightarrow N$ the bialgebroids $A$ and $B$ can be equipped with antipodes, making them dual Hopf algebroids.

It is important to emphasize that we use the term Hopf algebroid in the sense of [2] and not in the sense of [13]. Though - roughly speaking - both definitions can be summarized as a 'bialgebroid with antipode', the two definitions are not equivalent - as it is illustrated by an example in [2]. In [2] we give some equivalent definitions of the Hopf algebroid. These are all common in the feature that the axioms are formulated without reference to a particular section of the canonical projection $A \underset{Z}{\otimes} A \rightarrow A \underset{L}{\otimes} A$, which has played important role in the definition of [13. The most appropriate form of the definition for the purposes of this paper involves two, left and right bialgebroid structures on the same ring that are connected by the antipode. In Section 2 we cite the definition and some properties of the Hopf algebroid from [2].

A further step of generalization of the 'symmetry reconstruction problem' was done in [25] where the symmetry of abstract D2 extensions was studied. A 1-morphism $\iota$ in an additive bicategory $\mathcal{C}$ is an abstract extension if it possesses a left dual $\bar{\iota}$. In the original paper [25] the D2 property of $\iota$ was introduced as

i) the 1-morphism $(\iota \times \bar{\iota}) \times \iota$ is a direct summand in a finite direct sum of copies of $\iota$.

In later publications (see 11] for example) this property has been renamed as left D2 property. The condition that

ii) the 1-morphism $(\bar{\iota} \times \iota) \times \bar{\iota}$ is a direct summand in a finite direct sum of copies of $\bar{\iota}$ was called right D2 property. The 1-morphism $\iota$ is D2 in this later sense if both the left and right D2 conditions hold true. In this paper we use this latter terminology.

The name 'abstract extension' is motivated by the basic example: Observe that for a ring extension $N \rightarrow M$ the bimodule ${ }_{N} M_{M}$ is a 1-morphism in the bicategory of bimodules possessing a left dual ${ }_{M} M_{N}$. If the extension $N \rightarrow M$ is also D2 then the corresponding 1-morphism ${ }_{N} M_{M}$ satisfies the D2 condition. Hence a D2 ring extension determines a D2 1-morphism in an additive bicategory - called an abstract D2 extension.

Motivated by this example in 25] the 1-morphism $\iota$ in $\mathcal{C}$ possessing a left dual $\bar{\iota}$ is assumed to satisfy the left D2 condition. Then (denoting the horizontal composition in $\mathcal{C}$ by $\times)$ the ring $A:=\mathcal{C}^{2}(\iota \times \bar{\iota}, \iota \times \bar{\iota})$ of 2-endomorphisms is shown to carry a left bialgebroid structure. (The right D2 property of $\iota$ implies the existence of a right bialgebroid structure on $B:=\mathcal{C}^{2}(\bar{\iota} \times \iota, \bar{\iota} \times \iota)$.) The case when the finite index property and irreducibility of $\iota$ is required and $\mathcal{C}$ is semisimple and $k$-linear - leading to semisimple and cosemisimple Hopf algebra symmetry - is spelled out in detail in [14].

In this paper we generalize the analysis of the Section 3 of [2] to abstract extensions. That is we 
suppose that $\iota$ is a D2 Frobenius 1-morphism in an additive bicategory $\mathcal{C}$ with two sided dual $\bar{\iota}$. Under this assumption we show that the rings $A=\mathcal{C}^{2}(\iota \times \bar{\iota}, \iota \times \bar{\iota})$ and $B=\mathcal{C}^{2}(\bar{\iota} \times \iota, \bar{\iota} \times \iota)$ carry dual Hopf algebroid structures with non-degenerate two sided integrals in the sense of [2]. We also prove that a Hopf algebroid appears as a solution of this abstract symmetry problem if and only if it possesses a two sided non-degenerate integral.

It is discussed in 2, 1] that - in contrast to (weak) Hopf algebras - the Hopf algebroid structure on a given left bialgebroid is not unique. The choice of the antipode maps in Subsection [3.1] is a canonical construction fitting to the Frobenius structure of $\iota$. However, this canonical construction is responsible for the existence of the two sided non-degenerate integral.

This result raises the question how typical it is that we can find two sided non-degenerate integrals in Hopf algebroids. As a partial answer, it is proven in Proposition 5.13 in [2] that for any Hopf algebroid $\mathcal{A}$ possessing a non-degenerate left integral $\ell$ there exists another Hopf algebroid which is isomorphic to $\mathcal{A}$ as a left bialgebroid and in which $\ell$ is a two sided non-degenerate integral. In particular, it follows by combining the Proposition 5.13 in [2] with the Theorem 4.2 in [1] that for any finite dimensional (weak) Hopf algebra there exists a twisted antipode [6] leading to a Hopf algebroid with two sided non-degenerate integral. It is not known, however, whether any finite Hopf algebroid possesses a non-degenerate left integral, i.e. no generalization of the Larson-Sweedler theorem on bialgebroids is known.

The paper is organized as follows: In the Section 2 we shortly review some results from [24, 25, 11] on bialgebroids and from [2] on Hopf algebroids that we are going to use later on.

In the Subsection 3.1 we analyse the structures of the rings $A=\mathcal{C}^{2}(\iota \times \bar{\iota}, \iota \times \bar{\iota})$ and $B=\mathcal{C}^{2}(\bar{\iota} \times \iota, \bar{\iota} \times \iota)$ if only the Frobenius property of the 1-morphism $\iota$ in the additive bicategory $\mathcal{C}$ is assumed. Analogously to Section 3 of [2] already in this general situation - i.e. without assuming the D2 property - one can do 'Fourier analysis'. That is convolution products can be introduced both on $A$ and $B$ together with 'Fourier transformations' $A \rightarrow B$ relating the original and the convolution products. At this level of generality one can introduce the maps that are going to be the antipodes in the D2 case.

In the Subsection 3.2 we impose also the D2 condition on the 1-morphism $\iota$. Applying the results of 25] this implies the bialgebroid structures on $A$ and $B$. We prove that these bialgebroid structures together with the antipodes of Subsection 3.1] amount to dual Hopf algebroids in the sense of [2] both of them possessing two sided non-degenerate integrals.

In the Subsection 3.3 we address the question what Hopf algebroids appear as symmetries of absratct D2 Frobenius extensions. Being given a Hopf algebroid $\mathcal{A}$ possessing a two sided non-degenerate integral, we construct a D2 Frobenius 1-morphism $\mathcal{X}$ in the additive bicategory of internal bimodules in its module category. We show that the symmetry of $\mathcal{X}$ is described by $\mathcal{A}$. Combining this result with the one in Subsection 3.2 we conclude that the existence of a two sided non-degenerate integral [2] in the Hopf algebroid is a sufficient and necessary condition for it to appear as the symmetry of an abstract D2 Frobenius extension.

In the Appendix we sketch briefly the construction of the bicategory of internal bimodules in a monoidal category - used as a tool in Subsection 3.3

All rings appearing in the paper are associative and unital. The category of left/right/bi- modules over the ring $R$ is denoted by ${ }_{R} \mathcal{M} / \mathcal{M}_{R} /{ }_{R} \mathcal{M}_{R}$.

\section{Preliminaries: Bialgebroid and Hopf algebroid}

In this section we summarize our notations and the basic definitions of bialgebroids and Hopf algebroids that will be used later on. For more about bialgebroids we refer to the literature [28, 22, 5, 11, 23, 25, 26] and about Hopf algebroids to [2, 1].

The bialgebroid [13, 29, 25] or Takeuchi $\times_{L}$-bialgebra [28] is a generalization of the bialgebra to the case of a non-commutative base ring ring $L$ :

Definition 2.1 A left bialgebroid $\mathcal{A}_{L}$ consists of the data $\left(A, L, s_{L}, t_{L}, \gamma_{L}, \pi_{L}\right)$. The $A$ and $L$ are associative unital rings, the total and base rings, respectively. The $s_{L}: L \rightarrow A$ and $t_{L}: L^{o p} \rightarrow A$ are ring homomorphisms such that the images of $L$ under the source map $s_{L}$ and target map $t_{L}$ in $A$ commute making $A$ an $L-L$ bimodule:

$$
l \cdot a \cdot l^{\prime}:=s_{L}(l) t_{L}\left(l^{\prime}\right) a .
$$


The bimodule (2.1) is denoted by ${ }_{L} A_{L}$. The triple $\left({ }_{L} A_{L}, \gamma_{L}, \pi_{L}\right)$ is a comonoid in ${ }_{L} \mathcal{M}_{L}$. Introducing the Sweedler's notation $\gamma_{L}(a) \equiv a_{(1)} \otimes a_{(2)} \in A_{L} \otimes{ }_{L} A$ the identities

$$
\begin{aligned}
a_{(1)} t_{L}(l) \otimes a_{(2)} & =a_{(1)} \otimes a_{(2)} s_{L}(l) \\
\gamma_{L}\left(1_{A}\right) & =1_{A} \otimes 1_{A} \\
\gamma_{L}(a b) & =\gamma_{L}(a) \gamma_{L}(b) \\
\pi_{L}\left(1_{A}\right) & =1_{L} \\
\pi_{L}\left(a s_{L} \circ \pi_{L}(b)\right)= & \pi_{L}
\end{aligned}
$$

are required for all $l \in L$ and $a, b \in A$. The requirement (2.4) makes sense in view of (2.2).

If $\mathcal{A}_{L}=\left(A, L, s_{L}, t_{L}, \gamma_{L}, \pi_{L}\right)$ is a left bialgebroid then so is the co-opposite $\mathcal{A}_{L}$ cop $=\left(A, L^{o p}, t_{L}, s_{L}, \gamma_{L}^{o p}\right.$, $\left.\pi_{L}\right)$ where $\gamma_{L}^{o p}$ denotes the opposite coproduct $a \mapsto a_{(2)} \otimes a_{(1)}$. The opposite $\mathcal{A}_{L}^{o p}=\left(A^{o p}, L, t_{L}, s_{L}, \gamma_{L}, \pi_{L}\right)$ is a right bialgebroid in the sense of [11]:

Definition 2.2 A right bialgebroid $\mathcal{A}_{R}$ consists of the data $\left(A, R, s_{R}, t_{R}, \gamma_{R}, \pi_{R}\right)$. The $A$ and $R$ are associative unital rings, the total and base rings, respectively. The $s_{R}: R \rightarrow A$ and $t_{R}: R^{o p} \rightarrow A$ are ring homomorphisms such that the images of $R$ under the source map $s_{R}$ and target map $t_{R}$ in $A$ commute making $A$ an $R$ - $R$ bimodule:

$$
r \cdot a \cdot r^{\prime}:=a s_{R}\left(r^{\prime}\right) t_{R}(r) .
$$

The bimodule (2.7) is denoted by ${ }^{R} A^{R}$. The triple $\left({ }^{R} A^{R}, \gamma_{R}, \pi_{R}\right)$ is a comonoid in ${ }_{R} \mathcal{M}_{R}$. Introducing the Sweedler's notation $\gamma_{R}(a) \equiv a^{(1)} \otimes a^{(2)} \in A^{R} \otimes{ }^{R} A$ the identities

$$
\begin{aligned}
s_{R}(r) a^{(1)} \otimes a^{(2)} & =a^{(1)} \otimes t_{R}(r) a^{(2)} \\
\gamma_{R}\left(1_{A}\right) & =1_{A} \otimes 1_{A} \\
\gamma_{R}(a b) & =\gamma_{R}(a) \gamma_{R}(b) \\
\pi_{R}\left(1_{A}\right) & =1_{R} \\
\pi_{R}\left(s_{R} \circ \pi_{R}(a) b\right)= & \pi_{R} \quad(a b)=\pi_{R}\left(t_{R} \circ \pi_{R}(a) b\right)
\end{aligned}
$$

are required for all $r \in R$ and $a, b \in A$.

The $L$-actions of the bimodule ${ }_{L} A_{L}$ are given by left multiplication and the $R$-actions of the bimodule ${ }^{R} A^{R}$ are given by right multiplication. We can define further bimodules:

$$
\begin{array}{ll}
{ }^{L} A^{L}: & l \cdot a \cdot l^{\prime}:=a t_{L}(l) s_{L}\left(l^{\prime}\right) \\
{ }_{R} A_{R}: & r \cdot a \cdot r^{\prime}:=s_{R}(r) t_{R}\left(r^{\prime}\right) a .
\end{array}
$$

In the case of left/right bialgebroids the category $\left({ }_{A} \mathcal{M}, L, \mathbf{l}, \mathbf{r}, \mathbf{a}\right) /\left(\mathcal{M}_{A}, R, \mathbf{l}, \mathbf{r}, \mathbf{a}\right)$ of left/right $A$ modules has the monoidal structure inherited from the bimodule category ${ }_{L} \mathcal{M}_{L} /{ }_{R} \mathcal{M}_{R}$. For left/right $A$-modules $M$ and $N$ the $A$-module structure on $M \otimes_{L} N / M \otimes_{R} N$ is given by $a \cdot(m \otimes n):=a_{(1)} \cdot m \otimes$ $a_{(2)} \cdot n /(m \otimes n) \cdot a:=m \cdot a^{(1)} \otimes n \cdot a^{(2)}-$ for $a \in A, m \in M$ and $n \in N$. The monoidal unit is the left $A$-module on $L: a \cdot l:=\pi_{L}\left(a s_{L}(l)\right) /$ the right $A$-module on $R: r \cdot a:=\pi_{R}\left(s_{R}(r) a\right)$ - for $a \in A, l \in L$ and $r \in R$. The forgetful functor $\Phi_{L}:{ }_{A} \mathcal{M} \rightarrow{ }_{L} \mathcal{M}_{L} / \Phi_{R}: \mathcal{M}_{A} \rightarrow{ }_{R} \mathcal{M}_{R}$ is strong monoidal [25].

The homomorphisms of bialgebroids we use in this paper are the bialgebroid maps of [26]:

Definition 2.3 A left bialgebroid homomorphism $\mathcal{A}_{L} \rightarrow \mathcal{A}_{L}^{\prime}$ is a pair of ring homomorphisms $(\Phi: A \rightarrow$ $A^{\prime}, \phi: L \rightarrow L^{\prime}$ ) such that

$$
\begin{aligned}
s_{L}^{\prime} \circ \phi & =\Phi \circ s_{L} \\
t_{L}^{\prime} \circ \phi & =\Phi \circ t_{L} \\
\pi_{L}^{\prime} \circ \Phi & =\phi \circ \pi_{L} \\
\gamma_{L}^{\prime} \circ \Phi & =(\Phi \otimes \Phi) \circ \gamma_{L} .
\end{aligned}
$$


The last condition makes sense since by the first two conditions $\Phi \otimes \Phi$ is a well defined map $A_{L} \otimes{ }_{L} A \rightarrow$ $A_{L^{\prime}} \otimes_{L^{\prime}} A$. The pair $(\Phi, \phi)$ is an isomorphism of left bialgebroids if it is a left bialgebroid homomorphism such that both $\Phi$ and $\phi$ are bijective.

A right bialgebroid homomorphism (isomorphism) $\mathcal{A}_{R} \rightarrow \mathcal{A}_{R}^{\prime}$ is a left bialgebroid homomorphism (isomorphism) $\left(\mathcal{A}_{R}\right)^{o p} \rightarrow\left(\mathcal{A}_{R}^{\prime}\right)^{o p}$.

Let $\mathcal{A}_{L}$ be a left bialgebroid. The equation (2.1) describes two $L$-modules $A_{L}$ and ${ }_{L} A$. Their $L$-duals are the additive groups of $L$-module maps:

$$
\mathcal{A}_{*}:=\left\{\phi_{*}: A_{L} \rightarrow L_{L}\right\} \quad \text { and } \quad{ }_{*} \mathcal{A}:=\left\{{ }_{*} \phi:{ }_{L} A \rightarrow{ }_{L} L\right\}
$$

where ${ }_{L} L$ stands for the left regular and $L_{L}$ for the right regular $L$-module. Both $\mathcal{A}_{*}$ and ${ }_{*} \mathcal{A}$ carry left $A$ module structures via the transpose of the right regular action of $A$. For $\phi_{*} \in \mathcal{A}_{*},{ }_{*} \phi{ }_{*} \mathcal{A}$ and $a, b \in A$ we have:

$$
\left(a \rightarrow \phi_{*}\right)(b)=\phi_{*}(b a) \quad \text { and } \quad\left(a \neg_{*} \phi\right)(b)={ }_{*} \phi(b a) .
$$

Similarly, in the case of a right bialgebroid $\mathcal{A}_{R}$ - denoting the left and right regular $R$-modules by ${ }^{R} R$ and $R^{R}$, respectively, - the two $R$-dual additive groups

$$
\mathcal{A}^{*}:=\left\{\phi^{*}: A^{R} \rightarrow R^{R}\right\} \quad \text { and } \quad{ }^{*} \mathcal{A}:=\left\{{ }^{*} \phi:{ }^{R} A \rightarrow{ }^{R} R\right\}
$$

carry right $A$-module structures:

$$
\left(\phi^{*} \leftarrow a\right)(b)=\phi^{*}(a b) \quad \text { and } \quad\left({ }^{*} \phi-a\right)(b)={ }^{*} \phi(a b) .
$$

The comonoid structures can be transposed to give monoid (i.e. ring) structures to the duals. In the case of a left bialgebroid $\mathcal{A}_{L}$

$$
\left(\phi_{*} \psi_{*}\right)(a)=\psi_{*}\left(s_{L} \circ \phi_{*}\left(a_{(1)}\right) a_{(2)}\right) \quad \text { and } \quad\left({ }_{*} \phi_{*} \psi\right)(a)={ }_{*} \psi\left(t_{L} \circ{ }_{*} \phi\left(a_{(2)}\right) a_{(1)}\right)
$$

for ${ }_{*} \phi,{ }_{*} \psi \in{ }_{*} \mathcal{A}, \phi_{*}, \psi_{*} \in \mathcal{A}_{*}$ and $a \in A$.

Similarly, in the case of a right bialgebroid $\mathcal{A}_{R}$

$$
\left(\phi^{*} \psi^{*}\right)(a)=\phi^{*}\left(a^{(2)} t_{R} \circ \psi^{*}\left(a^{(1)}\right)\right) \quad \text { and } \quad\left({ }^{*} \phi^{*} \psi\right)(a)={ }^{*} \phi\left(a^{(1)} s_{R} \circ * \psi\left(a^{(2)}\right)\right)
$$

for $\phi^{*}, \psi^{*} \in \mathcal{A}^{*},{ }^{*} \phi,{ }^{*} \psi \in{ }^{*} \mathcal{A}$ and $a \in A$.

In the case of a left bialgebroid $\mathcal{A}_{L}$ also the ring $A$ has right $\mathcal{A}_{*^{-}}$and right ${ }_{*} \mathcal{A}$ - module sructures:

$$
a<\phi_{*}=s_{L} \circ \phi_{*}\left(a_{(1)}\right) a_{(2)} \quad \text { and } \quad a \leftarrow_{*} \phi=t_{L} \circ_{*} \phi\left(a_{(2)}\right) a_{(1)}
$$

for $\phi_{*} \in \mathcal{A}_{*},{ }_{*} \phi \in{ }_{*} \mathcal{A}$ and $a \in A$.

Similarly, in the case of a right bialgebroid $\mathcal{A}_{R}$ the ring $A$ has left $\mathcal{A}^{*}$ - and left ${ }^{*} \mathcal{A}$ structures:

$$
\phi^{*} \rightarrow a=a^{(2)} t_{R} \circ \phi^{*}\left(a^{(1)}\right) \quad \text { and } \quad * \rightarrow a=a^{(1)} s_{R} \circ^{*} \phi\left(a^{(2)}\right)
$$

for $\phi^{*} \in \mathcal{A}^{*},{ }^{*} \phi \in{ }^{*} \mathcal{A}$ and $a \in A$.

As it was proven in 11], if the $L(R)$ module structure on $A$ is finitely generated projective then the corresponding dual has also a bialgebroid structure.

The total ring of a Hopf algebroid carries eight canonical module structures over the base ring modules of the kind (2.1), (2.7), (2.8) and (2.9). In this situation the standard notation for the tensor product of modules, e.g. $A \underset{R}{\otimes} A$, would be ambigous. For this reason we put marks on both modules as in $A^{R} \otimes{ }^{R} A$, for example, to indicate the module structures taking part in the tensor products.

For coproduts of left bialgebroids we use the Sweedler notation in the form $\gamma_{L}(a)=a_{(1)} \otimes a_{(2)}$ and of right bialgebroids $\gamma_{R}(a)=a^{(1)} \otimes a^{(2)}$.

The Hopf algebroid introduced in 2] has both left and right bialgebroid structures and an antipode relating the two ${ }^{1}$ :

\footnotetext{
${ }^{1}$ Actually the original definition in $\left[2\right.$ was formulated in terms of the pair $\left(\mathcal{A}_{L}, S\right)$ consisting of a left bialgebroid and an antipode. It was proven that there exists a right bialgebroid $\mathcal{A}_{R}$ together with which the triple $\mathcal{A}=\left(\mathcal{A}_{L}, \mathcal{A}_{R}, S\right)$ satisfies the Definition 2.4 below. The right bialgebroid $\mathcal{A}_{R}$ is unique up to an irrelevant isomorphism.
} 
Definition 2.4 The triple $\mathcal{A}=\left(\mathcal{A}_{L}, \mathcal{A}_{R}, S\right)$ is a Hopf algebroid if $\mathcal{A}_{L}=\left(A, L, s_{L}, t_{L}, \gamma_{L}, \pi_{L}\right)$ is a left bialgebroid and $\mathcal{A}_{R}=\left(A, R, s_{R}, t_{R}, \gamma_{R}, \pi_{R}\right)$ is a right bialgebroid such that the base rings are related to each other via $R \simeq L^{o p}$ and

$$
\text { i) } s_{L}(L)=t_{R}(R) \quad t_{L}(L)=s_{R}(R)
$$

as subrings of $A$,

$$
\text { ii) } \begin{aligned}
\left(\gamma_{L} \otimes \mathrm{id}_{A}\right) \circ \gamma_{R} & =\left(\mathrm{id}_{A} \otimes \gamma_{R}\right) \circ \gamma_{L} \\
\left(\gamma_{R} \otimes \mathrm{id}_{A}\right) \circ \gamma_{L} & =\left(\mathrm{id}_{A} \otimes \gamma_{L}\right) \circ \gamma_{R}
\end{aligned}
$$

as maps $A \rightarrow A_{L} \otimes{ }_{L} A^{R} \otimes{ }^{R} A$ and $A \rightarrow A^{R} \otimes{ }^{R} A_{L} \otimes{ }_{L} A$, respectively.

The map $S: A \rightarrow A$ is a bijection of additive groups such that

$$
\text { iii) } S\left(t_{L}(l) a t_{L}\left(l^{\prime}\right)\right)=s_{L}\left(l^{\prime}\right) S(a) s_{L}(l) \quad S\left(t_{R}\left(r^{\prime}\right) a t_{R}(r)\right)=s_{R}(r) S(a) s_{R}\left(r^{\prime}\right)
$$

for all $l, l^{\prime} \in L, r, r^{\prime} \in R$ and $a \in A$. The requirement (2.14) makes the expressions $S\left(a_{(1)}\right) a_{(2)}$ and $a^{(1)} S\left(a^{(2)}\right)$ meaningful. The axioms

$$
\text { iv) } S\left(a_{(1)}\right) a_{(2)}=s_{R} \circ \pi_{R}(a) \quad a^{(1)} S\left(a^{(2)}\right)=s_{L} \circ \pi_{L}(a)
$$

are required for all $a$ in $A$.

We emphasize that this notion of Hopf algebroid is different from the one introduced in [13].

Definition 2.5 A Hopf algebroid homomorphism $\left(\mathcal{A}_{L}, \mathcal{A}_{R}, S\right) \rightarrow\left(\mathcal{A}_{L}^{\prime}, \mathcal{A}_{R}^{\prime}, S^{\prime}\right)$ is a left bialgebroid homomorphism $(\Phi, \phi): \mathcal{A}_{L} \rightarrow \mathcal{A}_{L}^{\prime}$.

Since it is proven in [2] Proposition 4.3 that both $\left(S, \pi_{R} \circ s_{L}\right)$ and $\left(S^{-1}, \pi_{R} \circ t_{L}\right)$ are left bialgebroid isomorphisms $\mathcal{A}_{L} \rightarrow\left(\mathcal{A}_{R}\right)_{\text {cop }}^{o p}$, for a Hopf algebroid homomorphism $(\Phi, \phi):\left(\mathcal{A}_{L}, \mathcal{A}_{R}, S\right) \rightarrow\left(\mathcal{A}_{L}^{\prime}, \mathcal{A}_{R}^{\prime}, S^{\prime}\right)$ both $\left(S^{\prime} \circ \Phi \circ S^{-1}, \pi_{R}^{\prime} \circ s_{L}^{\prime} \circ \phi \circ \pi_{L} \circ t_{R}\right)$ and $\left(S^{\prime-1} \circ \Phi \circ S, \pi_{R}^{\prime} \circ t_{L}^{\prime} \circ \phi \circ \pi_{L} \circ s_{R}\right)$ are right bialgebroid homomorphisms : $\mathcal{A}_{R} \rightarrow \mathcal{A}_{R}^{\prime}$.

A Hopf algebroid isomorphism is a Hopf algebroid homomorphism $(\Phi, \phi)$ such that both maps $\Phi$ and $\phi$ are bijective.

A Hopf algebroid homomorphism $(\Phi, \phi):\left(\mathcal{A}_{L}, \mathcal{A}_{R}, S\right) \rightarrow\left(\mathcal{A}_{L}^{\prime}, \mathcal{A}_{R}^{\prime}, S^{\prime}\right)$ is strict if $\Phi \circ S=S^{\prime} \circ \Phi$.

The existence of non-strict isomorphisms of Hopf algebroids is a new feature compared to (weak) Hopf algebras.

In the rest of this subsection let $\mathcal{A}=\left(\mathcal{A}_{L}, \mathcal{A}_{R}, S\right)$ be a Hopf algebroid where $\mathcal{A}_{L}=\left(A, L, s_{L}, t_{L}, \gamma_{L}, \pi_{L}\right)$ is the left bialgebroid and $\mathcal{A}_{R}=\left(A, R, s_{R}, t_{R}, \gamma_{R}, \pi_{R}\right)$ is the right bialgebroid underlying $\mathcal{A}$. The left and right integrals in a Hopf algebroid are introduced as the invariants of the left and right regular modules, respectively:

Definition 2.6 The left integrals are the elements of the right ideal:

$$
\mathcal{I}^{L}(\mathcal{A}):=\left\{\ell \in A \mid a \ell=s_{L} \circ \pi_{L}(a) \ell \quad \forall a \in A\right\} .
$$

The right integrals are the elements of the left ideal:

$$
\mathcal{I}^{R}(\mathcal{A}):=\left\{\Upsilon \in A \mid \Upsilon a=\Upsilon s_{R} \circ \pi_{R}(a) \quad \forall a \in A\right\} .
$$

The following lemma is cited from [2] Lemma 5.2 and it will be of importance in the considerations of this paper:

Lemma 2.7 The following characterizations of right/left integrals are equivalent:
i) $\Upsilon \in \mathcal{I}^{R}(\mathcal{A})$
ii) $\Upsilon a=\Upsilon t_{R} \circ \pi_{R}(a)$
iii) $\quad S(\Upsilon) \in \mathcal{I}^{L}(\mathcal{A})$
iv) $S^{-1}(\Upsilon) \in \mathcal{I}^{L}(\mathcal{A})$
v) $\left.\Upsilon_{(1)} a \otimes \Upsilon_{(2)}=\Upsilon_{(1)} \otimes \Upsilon_{(2)} S(a)\right)$ as elements of $A_{L} \otimes{ }_{L} A$

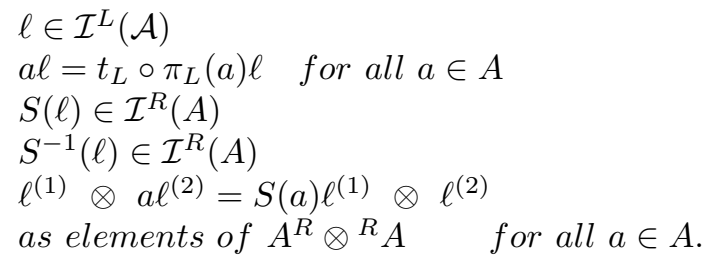


The left integral $\ell$ was called non-degenerate in [2] if the maps

$$
\begin{aligned}
& \ell_{R}: \mathcal{A}^{*} \rightarrow A \quad \phi^{*} \mapsto \phi^{*} \rightarrow \ell \quad \text { and } \\
& { }_{R} \ell:{ }^{*} \mathcal{A} \rightarrow A \quad{ }^{*} \quad \mapsto{ }^{*} \phi \rightarrow \ell
\end{aligned}
$$

are bijective. It follows that introducing $\lambda^{*}:=\ell_{R}^{-1}\left(1_{A}\right)$ the inverses of the maps $\ell_{R}$ and ${ }_{R} \ell$ are written as

$$
\ell_{R}^{-1}(a)=\lambda^{*} \leftarrow S(a) \quad{ }_{R} \ell^{-1}(a)=\lambda^{*} \circ S-S^{-1}(a) .
$$

Analogously, the right integral $\Upsilon$ is non-degenerate if the maps

$$
\begin{array}{llll}
{ }_{L} \Upsilon:{ }_{*} \mathcal{A} \rightarrow A & * \phi & \mapsto \Upsilon \leftarrow_{*} \phi & \text { and } \\
\Upsilon_{L}: \mathcal{A}_{*} \rightarrow A & \phi_{*} & \mapsto \Upsilon<\phi_{*}
\end{array}
$$

are bijective. Introducing ${ }_{*} \rho:={ }_{L} \Upsilon^{-1}\left(1_{A}\right)$ the inverses of the maps ${ }_{L} \Upsilon$ and $\Upsilon_{L}$ are written as

$$
\left.{ }_{L} \Upsilon^{-1}(a)=S(a) \rightarrow * \rho \quad \Upsilon_{L}^{-1}(a)=S^{-1}(a) \rightarrow{ }_{*} \rho \circ S\right) .
$$

It is proven in [2] Theorem 5.5 that the existence of a non-degenerate left integral $\ell$ in the Hopf algebroid $\mathcal{A}$ implies that all ring extensions $s_{L}: L \rightarrow A, t_{L}: L^{o p} \rightarrow A, s_{R}: R \rightarrow A$ and $t_{R}: R^{o p} \rightarrow A$ are Frobenius extensions. In particular the modules ${ }_{L} A, A_{L}, A^{R}$ and ${ }^{R} A$ are finitely generated projective hence all rings ${ }_{*} \mathcal{A}, \mathcal{A}_{*} \mathcal{A}^{*}$ and ${ }^{*} \mathcal{A}$ carry bialgebroid structures. The right bialgebroids ${ }_{*} \mathcal{A}_{R}$ and $\mathcal{A}_{* R}$ and also the left bialgebroids $\mathcal{A}^{*}{ }_{L}$ and ${ }^{*} \mathcal{A}_{L}$ were shown to be isomorphic. Furthermore, the left bialgebroid $\mathcal{A}^{*}{ }_{L}$ and the right bialgebroid ${ }_{*} \mathcal{A}_{R}$ turned out to be anti-isomorphic.

What is more, fixing a non-degenerate left integral $\ell$ in $\mathcal{A}$, one can construct an ( $\ell$-dependent) Hopf algebroid structure $\mathcal{A}^{*} \ell$ on the ring $\mathcal{A}^{*}$ with two sided non-degenerate integral $\ell_{R}^{-1}\left(1_{A}\right)$.

The Hopf algebroids $\mathcal{A}^{*} \ell$ and $\mathcal{A}^{*} \ell^{\prime}$ - corresponding to different choices of the non-degenerate left integral - are isomorphic but not strictly isomorphic.

Assuming the existence of a two sided (i.e. both left and right) non-degenerate integral $i^{2}$, the Hopf algebroid $\mathcal{A}^{*}{ }_{i}$ can be interpreted as the dual Hopf algebroid of $\mathcal{A}$ in the following sense:

Theorem 2.8 [2] Let $\mathcal{A}$ be a Hopf algebroid with two sided non-degenerate integral $i$. Then the following data define a Hopf algebroid $\mathcal{A}^{*}$ : The left bialgebroid over the base $R$ and the right bialgebroid over the base $L$ on the ring $\mathcal{A}^{*}$ given by

$$
\begin{array}{ll}
s_{L}^{*}(r)(a)=r \pi_{R}(a) & s_{R}^{*}(l)(a)=\pi_{R}\left(s_{L}(l) a\right) \\
t_{L}^{*}(r)(a)=\pi_{R}\left(s_{R}(r) a\right) & t_{R}^{*}(l)(a)=\lambda^{*}\left(S\left(i t_{L}(l)\right) a\right) \\
\gamma_{L}^{*}\left(\phi^{*}\right)=\phi^{*} \leftarrow i^{(1)} \otimes i_{R}{ }^{-1}\left(i^{(2)}\right) & \gamma_{R}^{*}\left(\phi^{*}\right)=\phi^{*} \leftarrow S^{2}\left(i^{(2)}\right) \otimes i_{R}{ }^{-1}\left(i^{(1)}\right) \\
\pi_{L}^{*}\left(\phi^{*}\right)=\phi^{*}\left(1_{A}\right) & \pi_{R}^{*}\left(\phi^{*}\right)=\pi_{L} \circ s_{R} \circ\left(\lambda^{*} \phi^{*}\right)(i)
\end{array}
$$

where $\lambda^{*}=i_{R}{ }^{-1}\left(1_{A}\right)$, and the antipode $S^{*} \equiv i_{R}{ }^{-1} \circ S \circ i_{R}: \mathcal{A}^{*} \rightarrow \mathcal{A}^{*}$. By iterating the construction the second dual Hopf algebroid $\left(\mathcal{A}^{*}{ }_{i}\right)_{i_{R}^{-1}\left(1_{A}\right)}^{*}$ is strictly isomorphic to $\mathcal{A}$.

The above Theorem 2.8] is a variant of [2] Theorem 5.17.

\section{Hopf algebroid symmetry of abstract D2 Frobenius extensions}

\subsection{Some harmonic analysis}

We adopt a graphical notation for bicategories using planar diagrams similar to that has been used e.g. by Yetter for sovereign monoidal categories in 32. The only difference is that now also the planar regions carry labels, the objects of the bicategory. As an experiment, we will also employ a notation for the coherence isomorphisms at the price of introducing some metrical information into the diagrams which, of course, destroys the topological nature of 2-categorical diagrams.

\footnotetext{
${ }^{2}$ For a two sided integral $i$ the non-degeneracy as a left and as a right integral are equivalent.
} 
Let $\left(\mathcal{C}, s_{0}, t_{0}, s_{1}, t_{1}, \circ, \times, \mathbf{l}, \mathbf{r}, \mathbf{a}\right)$ be a bicategory. We use the following graphical rules. For a 1-cell $a$ we draw a vertical line. The area right to the line is labelled by the source 0-cell $s_{0}(a)$ and the area left to the line by the target $t_{0}(a)$. For the 2-cell $x$ we draw a box with upper 'leg' its source $s_{1}(x)$ and lower 'leg' the target $t_{1}(x)$. The vertical composition $x \circ y$ is represented by a picture in which $x$ is placed under $y$. The horizontal composition $x \times y$ is represented by a picture in which $x$ is placed left to $y$. Since the horizontal composition is not strictly associative we take care about the horizontal distances between two lines or boxes. The successive bracketing is represented by growing distances. For the coherence natural isomorphisms we use the graphical notation

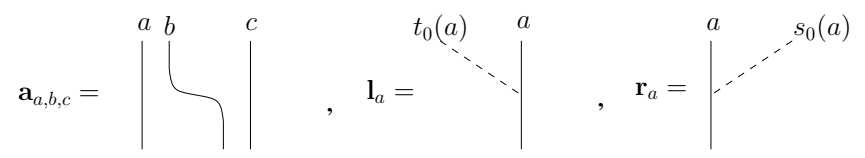

where the dashed line emphasizes that we have a 0 -cell here.

Let $\iota$ be a Frobenius 1-morphism of $\mathcal{C}$ - that is suppose that $\iota$ has a two sided dual $\bar{\iota}$. The Frobenius property means the existence of 2 -morphisms

$$
\begin{array}{ll}
\mathrm{ev}_{R} \in \mathcal{C}^{2}\left(\iota \times \bar{\iota}, t_{0}(\iota)\right) & \operatorname{coev}_{R} \in \mathcal{C}^{2}\left(s_{0}(\iota), \bar{\iota} \times \iota\right) \\
\mathrm{ev}_{L} \in \mathcal{C}^{2}\left(\bar{\iota} \times \iota, s_{0}(\iota)\right) & \operatorname{coev}_{R} \in \mathcal{C}^{2}\left(t_{0}(\iota), \iota \times \bar{\iota}\right)
\end{array}
$$

satisfying the relations

$$
\begin{aligned}
& \mathbf{r}_{\iota} \circ\left(\iota \times \mathrm{ev}_{L}\right) \circ \mathbf{a}_{\iota, \bar{\iota}, \iota} \circ\left(\operatorname{coev}_{L} \times \iota\right) \circ \mathbf{l}_{\iota}^{-1}=\iota \\
& \mathbf{l}_{\bar{\iota}} \circ\left(\mathrm{ev}_{L} \times \bar{\iota}\right) \circ \mathbf{a}_{\bar{\iota}, \iota, \bar{\iota}}^{-1} \circ\left(\bar{\iota} \times \operatorname{coev}_{L}\right) \circ \mathbf{r}_{\bar{\iota}}^{-1}=\bar{\iota} \\
& \mathbf{l}_{\iota} \circ\left(\operatorname{ev}_{R} \times \iota\right) \circ \mathbf{a}_{\iota, \bar{t}, \iota}^{-1} \circ\left(\iota \times \operatorname{coev}_{R}\right) \circ \mathbf{r}_{\iota}^{-1}=\iota \\
& \mathbf{r}_{\bar{\iota}} \circ\left(\bar{\iota} \times \operatorname{ev}_{R}\right) \circ \mathbf{a}_{\bar{\iota}, \iota, \bar{L}} \circ\left(\operatorname{coev}_{R} \times \bar{\iota}\right) \circ \mathbf{l}_{\bar{\iota}}^{-1}=\bar{\iota} .
\end{aligned}
$$

In the graphical notation we draw a vertical line directed downwards for $\iota$, upwards for $\bar{\iota}$ and
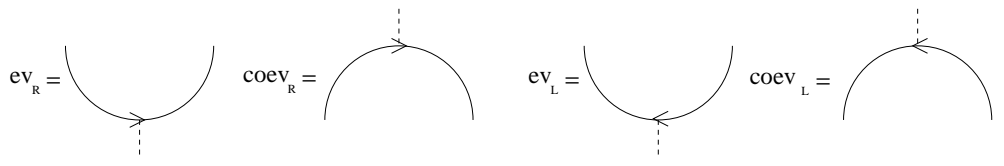

In this language the relations (3.2) read as
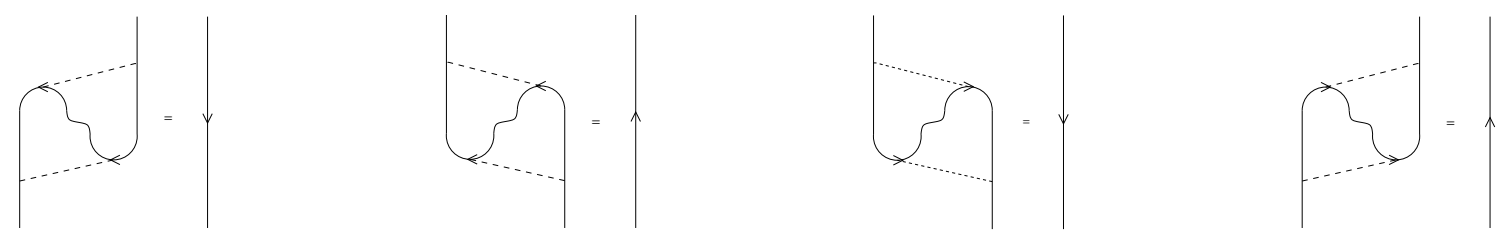

It follows from coherence that the meanderings of the lines as well as the dashed lines may be forgotten if equality of two 2-cells is to be proven graphically.

From now on we assume that the bicategory $\mathcal{C}$ is enriched over $\mathbf{A b}$, i.e., the hom sets are Abelian groups, written additively, and both the vertical and the horizontal compositions are group homomorphisms. In this situation both isomorphic additive groups $\mathrm{A}:=\mathcal{C}^{2}(\iota \times \bar{\iota}, \iota \times \bar{\iota})$ and $\mathrm{B}:=\mathcal{C}^{2}(\bar{\iota} \times \iota, \bar{\iota} \times \iota)$ carry two ring structures. The multiplication in $A:=(\mathrm{A}, \circ)$ and $B:=(\mathrm{B}, \mathrm{\circ})$ is given by the vertical 
composition $\circ$ of $\mathcal{C}$ while $\hat{A}:=(\mathrm{A}, *)$ and $\hat{B}:=(\mathrm{B}, *)$ can be defined - by fixing the 2-morphisms (3.1) as

$$
\begin{aligned}
a_{1} * a_{2}= & \left(\mathbf{r}_{\iota} \times \bar{\iota}\right) \circ\left[\left(\iota \times \mathrm{ev}_{L}\right) \times \bar{\iota}\right] \circ\left(\mathbf{a}_{\iota, \bar{L}, \iota} \times \bar{\iota}\right) \circ \mathbf{a}_{\iota \times \bar{\iota}, \iota, \bar{\iota}}^{-1} \circ\left(a_{1} \times a_{2}\right) \circ \\
\circ & \mathbf{a}_{\iota \times \bar{\iota}, \iota, \bar{\iota}} \circ\left(\mathbf{a}_{\iota, \bar{L}, \iota}^{-1} \times \bar{\iota}\right) \circ\left[\left(\iota \times \operatorname{coev}_{R}\right) \times \bar{\iota}\right] \circ\left(\mathbf{r}_{\iota}^{-1} \times \bar{\iota}\right) \\
b_{1} * b_{2}= & \left(\bar{\iota} \times \mathbf{l}_{\iota}\right) \circ\left[\bar{\iota} \times\left(\operatorname{ev}_{R} \times \iota\right)\right] \circ\left(\bar{\iota} \times \mathbf{a}_{\iota, \bar{\iota}, \iota}^{-1}\right) \circ \mathbf{a}_{\bar{\iota}, \iota, \bar{\iota} \times \iota \iota} \circ\left(b_{1} \times b_{2}\right) \circ \\
\circ & \mathbf{a}_{\bar{\iota}, \iota, \bar{\iota} \times \iota}^{-1} \circ\left(\bar{\iota} \times \mathbf{a}_{\iota, \bar{L}, \iota}\right) \circ\left[\bar{\iota} \times\left(\operatorname{coev}_{L} \times \iota\right)\right] \circ\left(\bar{\iota} \times \mathbf{l}_{\iota}^{-1}\right) .
\end{aligned}
$$

In the graphical language
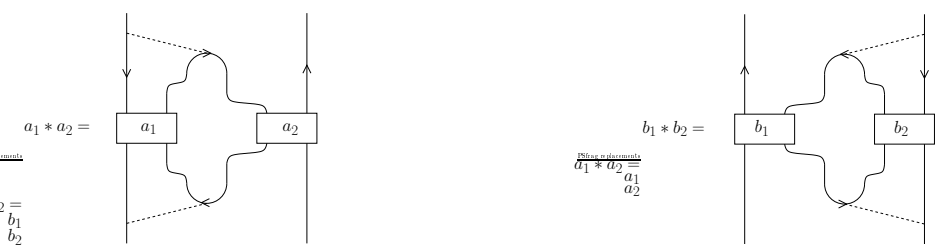

By coherence $\hat{A}$ and $\hat{B}$ are associative with units $i_{A}:=\operatorname{coev}_{L} \circ \mathrm{ev}_{R}$ and $i_{B}:=\operatorname{coev}_{R} \circ \mathrm{ev}_{L}$, respectively. This way we have four associative unital rings $A, B, \hat{A}$ and $\hat{B}$. We claim that $A$ is isomorphic to $\hat{B}$ and $B$ is isomorphic to $\hat{A}$. Let us define the Fourier transformations

$$
\begin{aligned}
& \mathcal{F}: \mathrm{A} \rightarrow \mathrm{B} \quad a \mapsto \mathbf{r}_{\bar{\iota} \times \iota} \circ\left[(\bar{\iota} \times \iota) \times \mathrm{ev}_{L}\right] \circ \mathbf{a}_{\bar{\iota} \times \iota, \bar{c}, \iota} \circ\left(\mathbf{a}_{\bar{l}, \iota, \bar{\iota}}^{-1} \times \iota\right) \circ[(\bar{\iota} \times a) \times \iota] \circ \\
& \left(\mathbf{a}_{\bar{\iota}, \iota, \bar{\iota}} \times \iota\right) \circ \mathbf{a}_{\bar{\imath} \times \iota, \bar{L}, \iota}^{-1} \circ\left[\operatorname{coev}_{R} \times(\bar{\iota} \times \iota)\right] \circ \mathbf{l}_{\bar{\iota} \times \iota}^{-1} \\
& \dot{\mathcal{F}}: \mathrm{A} \quad \rightarrow \mathrm{B} \quad a \mapsto \mathbf{l}_{\bar{\iota} \times \iota} \circ\left[\mathrm{ev}_{L} \times(\bar{\iota} \times \iota)\right] \circ \mathbf{a}_{\bar{l}, \iota, \bar{L} \times \iota}^{-1} \circ\left(\bar{\iota} \times \mathbf{a}_{\iota, \bar{L}, \iota}\right) \circ[\bar{\iota} \times(a \times \iota)] \circ \\
& \left(\bar{\iota} \times \mathbf{a}_{\iota, \bar{L}, \iota}^{-1}\right) \circ \mathbf{a}_{\bar{\iota}, \iota, \bar{L} \times \iota} \circ\left[(\bar{\iota} \times \iota) \times \operatorname{coev}_{R}\right] \circ \mathbf{r}_{\bar{\iota} \times \iota}^{-1}
\end{aligned}
$$

In picture:
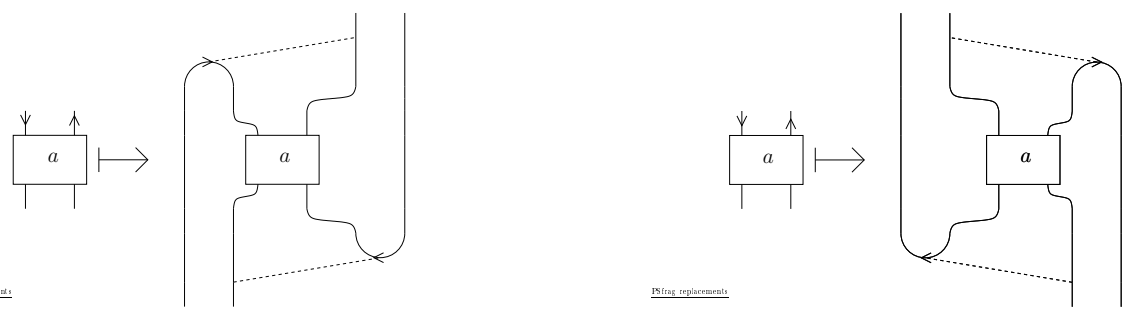

Both $\mathcal{F}$ and $\dot{\mathcal{F}}$ are bijections with inverses

$$
\begin{aligned}
& \mathcal{F}^{-1}: \mathrm{B} \rightarrow \mathrm{A} \quad b \mapsto \mathbf{1}_{\iota \times \bar{\iota}} \circ\left[\operatorname{ev}_{R} \times(\iota \times \bar{\iota})\right] \circ \mathbf{a}_{\iota, \bar{L}, \iota \times \bar{\iota}}^{-1} \circ\left(\iota \times \mathbf{a}_{\bar{\iota}, \iota, \bar{\iota}}\right) \circ[\iota \times(b \times \bar{\iota})] \circ \\
& \left.\left(\iota \times \mathbf{a}_{\bar{\imath}, \iota, \bar{l}}^{-1}\right) \circ \mathbf{a}_{\iota, \bar{L}, \iota \times \bar{\iota}} \circ[(\iota \times \bar{\iota})] \times \operatorname{coev}_{L}\right] \circ \mathbf{r}_{\iota \times \bar{\iota}}^{-1} \\
& \dot{\mathcal{F}}^{-1}: \mathrm{B} \rightarrow \mathrm{A} \quad b \mapsto \mathbf{r}_{\iota \times \bar{\iota}} \circ\left[(\iota \times \bar{\iota}) \times \mathrm{ev}_{R}\right] \circ \mathbf{a}_{\iota \times \bar{\iota}, \iota, \bar{l}} \circ\left(\mathbf{a}_{\iota, \bar{L}, \iota}^{-1} \times \bar{l}\right) \circ[(\iota \times b) \times \bar{l}] \circ \\
& \left(\mathbf{a}_{\iota, \bar{L}, \iota} \times \bar{\iota}\right) \circ \mathbf{a}_{\iota \times \bar{L}, \iota, \bar{\iota}}^{-1} \circ\left[\operatorname{coev}_{L} \times(\iota \times \bar{l})\right] \circ \mathbf{1}_{\iota \times \bar{\iota}}^{-1}
\end{aligned}
$$

They relate the products $\circ$ and $*$ as follows:

$$
\begin{aligned}
\mathcal{F}\left(a_{1} \circ a_{2}\right) & =\mathcal{F}\left(a_{2}\right) * \mathcal{F}\left(a_{1}\right) \\
\dot{\mathcal{F}}\left(a_{1} \circ a_{2}\right) & =\dot{\mathcal{F}}\left(a_{1}\right) * \dot{\mathcal{F}}\left(a_{2}\right) \\
\mathcal{F}\left(a_{1} * a_{2}\right) & =\mathcal{F}\left(a_{1}\right) \circ \mathcal{F}\left(a_{2}\right) \\
\dot{\mathcal{F}}\left(a_{1} * a_{2}\right) & =\dot{\mathcal{F}}\left(a_{2}\right) \circ \dot{\mathcal{F}}\left(a_{1}\right) .
\end{aligned}
$$

The equations (3.7) imply that the differences of the Fourier transformations give ring anti-automorphisms

$$
S_{A}:=\dot{\mathcal{F}}^{-1} \circ \mathcal{F}: A^{o p} \rightarrow A \text { and } S_{B}:=\mathcal{F} \circ \dot{\mathcal{F}}^{-1}: B^{o p} \rightarrow B .
$$


They are to be the antipodes in the case when $\iota$ satisfies the D2 condition discussed in the next subsection.

Let us investigate some bimodule map properties of the maps $S_{A}$ and $S_{B}$. Both additive groups A and B carry four commuting actions of the anti-isomorphic rings $L:=\mathcal{C}^{2}(\iota, \iota)$ and $R:=\mathcal{C}^{2}(\bar{\iota}, \bar{\iota})$ - given by 'composition on the four legs'. That is we have the ring isomorphisms

$$
\begin{array}{llll}
\mu: L & \rightarrow R^{o p} & l & \mapsto \mathbf{l}_{\bar{\iota}} \circ\left(\mathrm{ev}_{L} \times \bar{\iota}\right) \circ[(\bar{\iota} \times l) \times \bar{\iota}] \circ \mathbf{a}_{\bar{\iota}, l, \bar{\iota}}^{-1} \circ\left(\bar{\iota} \times \operatorname{coev}_{L}\right) \circ \mathbf{r}_{\bar{\iota}}^{-1} \\
\nu: L & \rightarrow R^{o p} \quad l & \mapsto \mathbf{r}_{\bar{\iota}} \circ\left(\bar{\iota} \times \mathrm{ev}_{R}\right) \circ[\bar{\iota} \times(l \times \bar{\iota})] \circ \mathbf{a}_{\bar{\iota}, \iota, \bar{L}} \circ\left(\operatorname{coev}_{R} \times \bar{\iota}\right) \circ \mathbf{l}_{\bar{\iota}}^{-1}
\end{array}
$$

which read in the graphical language as
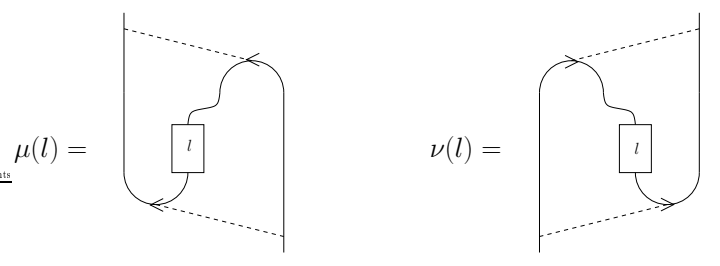

With the help of the maps $\mu$ and $\nu$ we can define the bimodules ${ }_{L} A_{L}$ and ${ }^{R} A^{R}$ as follows:

$$
\begin{array}{lll}
l \cdot a=(l \times \bar{\iota}) \circ a & a \cdot l & =(\iota \times \mu(l)) \circ a \\
a \cdot r=a \circ(\iota \times r) & r \cdot a & =a \circ\left(\nu^{-1}(r) \times \bar{\iota}\right) .
\end{array}
$$

Since

$$
\begin{array}{rll}
\mathcal{F}(l \cdot a)=(\bar{\iota} \times l) \circ \mathcal{F}(a) & \mathcal{F}(a \cdot l) & =\mathcal{F}(a) \circ(\bar{\iota} \times l) \\
\mathcal{F}(a \cdot r)=\mathcal{F}(a) \circ(r \times \iota) & \mathcal{F}(r \cdot a) & =(r \times \iota) \circ \mathcal{F}(a)
\end{array}
$$

and

$$
\begin{aligned}
\dot{\mathcal{F}}(l \cdot a) & =\dot{\mathcal{F}}(a) \circ(\mu(l) \times \iota) & \dot{\mathcal{F}}(a \cdot l) & =(\mu(l) \times \iota) \circ \dot{\mathcal{F}}(a) \\
\dot{\mathcal{F}}(a \cdot r) & =\left(\bar{\iota} \times \nu^{-1}(r)\right) \circ \dot{\mathcal{F}}(a) & \dot{\mathcal{F}}(r \cdot a) & =\dot{\mathcal{F}}(a) \circ\left(\bar{\iota} \times \nu^{-1}(r)\right)
\end{aligned}
$$

the map $S_{A}$ is compatible with the bimodule structures (3.10) that is it is a twisted bimodule map 2 ] ${ }_{L} A_{L} \rightarrow{ }^{R} A^{R}$ and also ${ }^{R} A^{R} \rightarrow{ }_{L} A_{L}:$

$$
S_{A}\left(l \cdot a \cdot l^{\prime}\right)=\nu\left(l^{\prime}\right) \cdot a \cdot \nu(l) \quad S_{A}\left(r \cdot a \cdot r^{\prime}\right)=\mu^{-1}\left(r^{\prime}\right) \cdot a \cdot \mu^{-1}(r) .
$$

\subsection{The depth 2 case}

The notion of depth 2 or shortly D2 property in the context of bicategories was introduced in [25] ${ }^{3}$ : Let $\mathcal{C}$ be an $\mathbf{A b}$-enriched bicategory closed under direct sums of 1-morphisms and possessing zero 1morphisms for any pair of objects. Such bicategories will be called additive. Let $\iota$ be a 1-morphism in $\mathcal{C}$ possessing a left dual $\bar{\iota}$. Then $\iota$ is said to satisfy the left D2 condition if $(\iota \times \bar{\iota}) \times \iota$ is a direct summand in a finite direct sum of $\iota$ 's. In this case - under the additional assumption that $s_{0}(\iota)$ is a direct summand in $\bar{\iota} \times \iota$, which can be relaxed - it was proven in 25 that for such a 1-morphism $\iota$ the ring $A=\left(\mathcal{C}^{2}(\iota \times \bar{\iota}, \iota \times \bar{\iota}), \circ\right)$ has a canonical left bialgebroid structure over the base $L=\mathcal{C}^{2}(\iota, \iota)$.

It is clear that if $(\bar{\iota} \times \iota) \times \bar{\iota}$ is a direct summand in a finite direct sum of $\bar{\iota}$ 's (right D2 condition) then the ring $B:=\left(\mathcal{C}^{2}(\bar{\iota} \times \iota, \bar{\iota} \times \iota), \circ\right)$ has a right bialgebroid structure.

In the sequel we will see that if $\iota$ is a D2 Frobenius 1-morphism

then so is $\bar{\iota}$. In this case the rings $A=\left(\mathcal{C}^{2}(\iota \times \bar{\iota}, \iota \times \bar{\iota}), \circ\right)$ and $B=\left(\mathcal{C}^{2}(\bar{\iota} \times \iota, \bar{\iota} \times \iota), \circ\right)$ carry left as well as right bialgebroid structures such that together with the antipodes $S_{A}$ and $S_{B}$ in (3.8) they are dual Hopf algebroids in the sense of [2].

Throughout the subsection let $\left(\mathcal{C}, s_{0}, t_{0}, s_{1}, t_{1}, \circ, \times, \mathbf{l}, \mathbf{r}, \mathbf{a}\right)$ be an additive bicategory. Let $\iota$ be a Frobenius 1-morphism in $\mathcal{C}$. In this case one can reformulate the $\mathrm{D} 2$ condition as follows:

\footnotetext{
${ }^{3}$ Recall that the terminology of $[25$ is somewhat different from the later publications. The condition that was called D2 property in 25 we call left D2 property, as it is explaned in the Introduction.
} 
Proposition 3.1 The $\iota$ satisfies the left D2 condition if and only if there exists an element $\sum_{i} y_{i} \otimes x_{i} \in$ $A^{L} \otimes{ }_{L} A$ such that

$$
\left(y_{i} \times \iota\right) \circ \mathbf{a}_{\iota, \bar{L}, \iota}^{-1} \circ\left(\iota \times \operatorname{coev}_{R}\right) \circ\left(\iota \times \mathrm{ev}_{L}\right) \circ \mathbf{a}_{\iota, \bar{t}, \iota} \circ\left(x_{i} \otimes \iota\right)=(\iota \times \bar{\iota}) \times \iota
$$

where $A^{L}$ is the right $L$ module defined as $a \cdot l:=a \circ(l \times \bar{\iota})$. In the graphical notation 3.14) reads as

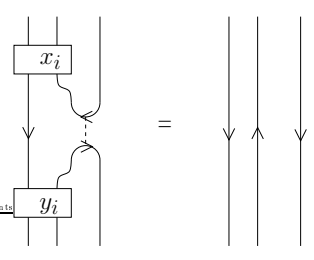

The element $\sum_{i} y_{i} \otimes x_{i} \in A^{L} \otimes{ }_{L} A$ is called a $\mathrm{D} 2$ quasi-basis for $\iota$.

Proof: The property that $(\iota \times \bar{\iota}) \times \iota$ is a direct summand in a finite direct sum of $\iota$ 's means the existence of finite sets of 2-cells

$$
\begin{aligned}
& \left\{\beta_{i}\right\}_{i=1 \ldots n} \subset \mathcal{C}^{2}((\iota \times \bar{\iota}) \times \iota, \iota) \\
& \left\{\beta_{i}^{\prime}\right\}_{i=1 \ldots n} \subset \mathcal{C}^{2}(\iota,(\iota \times \bar{\iota}) \times \iota)
\end{aligned}
$$

satisfying $\sum_{i} \beta_{i}^{\prime} \circ \beta_{i}=(\iota \times \bar{\iota}) \times \iota$. By the Frobenius property of $\iota$ this is further equivalent to the existence of the sets

$$
\begin{aligned}
& \left\{x_{i}:=\left(\beta_{i} \times \bar{\iota}\right) \circ \mathbf{a}_{\iota \times \bar{\iota}, \iota, \bar{\iota}}^{-1} \circ\left[(\iota \times \bar{\iota}) \times \operatorname{coev}_{L}\right] \circ \mathbf{r}_{\iota \times \bar{\iota}}^{-1}\right\}_{i=1 \ldots n} \quad \subset \quad \mathcal{C}^{2}(\iota \times \bar{\iota}, \iota \times \bar{\iota}) \\
& \left\{y_{i}:=\mathbf{r}_{\iota \times \bar{\iota}} \circ\left[(\iota \times \bar{\iota}) \times \mathrm{ev}_{R}\right] \circ \mathbf{a}_{\iota \times \bar{\iota}, \iota, \bar{\iota}} \circ\left(\beta_{i}^{\prime} \times \bar{\iota}\right)\right\}_{i=1 \ldots n} \subset \mathcal{C}^{2}(\iota \times \bar{\iota}, \iota \times \bar{\iota})
\end{aligned}
$$
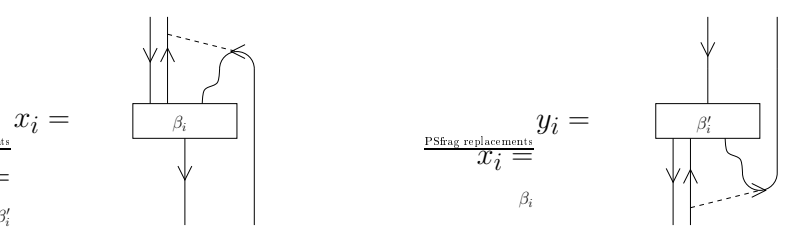

satisfying (3.14).

If $\sum_{i} y_{i} \otimes x_{i}$ is a D2 quasi-basis for $\iota$ then $\sum_{i} \mathcal{F} \circ \dot{\mathcal{F}}^{-1} \circ \mathcal{F}\left(x_{i}\right) \otimes \mathcal{F}\left(y_{i}\right) \in B^{R}{ }_{R}^{\otimes} B$ - where ${ }_{R} B$ is the left $R$-module defined as $r \cdot b:=(r \times \iota) \circ b$ and $B^{R}$ is the right $R$-module $b \cdot r=b \circ(r \times \iota)$ - is easily checked to be a D2 quasi-basis for $\bar{\iota}$. Since, owing to the Frobenius property of $\iota$, the right D2 condition on $\iota$ coincides with the left D2 condition on $\bar{\iota}$ we conclude that $\iota$ is D2 if and only if it is left D2 and if and only if $\iota$ is D2.

From now on let $\iota$ be a D2 Frobenius 1-morphism in $\mathcal{C}$ with D2 quasi-basis $y_{i} \otimes x_{i}$. We omit the summation symbol for summing over the D2 quasi-basis. The next Proposition shows that $y_{i} \otimes x_{i}$ is really a quasi-basis in the sense of [33]:

Proposition 3.2 The map

$$
s_{L}^{A}: L \rightarrow A \quad l \mapsto l \times \bar{\iota}
$$

is a Frobenius extension.

Proof: We construct the Frobenius map

$$
\phi_{L}: A \rightarrow L \quad a \mapsto \mathbf{r}_{\iota} \circ\left(\iota \times \operatorname{ev}_{L}\right) \circ \mathbf{a}_{\iota, \bar{c}, \iota} \circ(a \times \iota) \circ \mathbf{a}_{\iota, \bar{l}, \iota}^{-1} \circ\left(\iota \times \operatorname{coev}_{R}\right) \circ \mathbf{r}_{\iota}^{-1}
$$




$$
\phi_{L}(a)=
$$

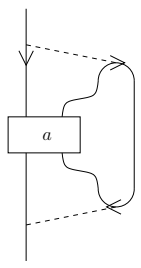

and show that it has the quasi basis $y_{i} \otimes x_{i}$. Making use of the coherence axioms in $\mathcal{C}$ and the relations (3.2) and (3.14) we have

$$
\begin{aligned}
& s_{L}^{A} \circ \phi_{L}\left(a \circ y_{i}\right) \circ x_{i}=\left(\mathbf{r}_{\iota} \times \bar{\iota}\right) \circ\left[\left(\iota \times \mathrm{ev}_{L}\right) \times \bar{\iota}\right] \circ\left(\mathbf{a}_{\iota, \bar{L}, \iota} \times \bar{\iota}\right) \circ[(a \times \iota) \times \bar{\iota}] \circ\left[\left(y_{i} \times \iota\right) \times \bar{\iota}\right] \circ\left(\mathbf{a}_{\iota, \bar{l}, \iota}^{-1} \times \bar{l}\right) \circ \\
& {\left[\left(\iota \times \operatorname{coev}_{R}\right) \times \bar{\imath}\right] \circ\left(\mathbf{r}_{\iota}^{-1} \times \bar{\iota}\right) \circ x_{i}=} \\
& =\left(\mathbf{r}_{\iota} \times \bar{\iota}\right) \circ\left[\left(\iota \times \mathrm{ev}_{L}\right) \times \bar{\iota}\right] \circ\left(\mathbf{a}_{\iota, \bar{l}, \iota} \times \bar{\iota}\right) \circ[(a \times \iota) \times \bar{\iota}] \circ\left[\left(y_{i} \times \iota\right) \times \bar{\iota}\right] \circ\left(\mathbf{a}_{\iota, \bar{L}, \iota}^{-1} \times \bar{l}\right) \circ \\
& {\left[\left(\iota \times \operatorname{coev}_{R}\right) \times \bar{\iota}\right] \circ\left(\mathbf{r}_{\iota}^{-1} \times \bar{\iota}\right) \circ\left(\iota \times \mathbf{l}_{\bar{\iota}}\right) \circ\left[\iota \times\left(\mathrm{ev}_{L} \times \bar{\iota}\right)\right] \circ\left(\iota \times \mathbf{a}_{\bar{\iota}, \iota, \bar{l}}^{-1}\right) \circ} \\
& {\left[\iota \times\left(\bar{\iota} \times \operatorname{coev}_{L}\right)\right] \circ\left(\iota \times \mathbf{r}_{\bar{L}}^{-1}\right) \circ x_{i}=} \\
& =\left(\mathbf{r}_{\iota} \times \bar{\iota}\right) \circ\left[\left(\iota \times \mathrm{ev}_{L}\right) \times \bar{\iota}\right] \circ\left(\mathbf{a}_{\iota, \bar{l}, \iota} \times \bar{\iota}\right) \circ[(a \times \iota) \times \bar{\iota}] \circ\left[\left(y_{i} \times \iota\right) \times \bar{\iota}\right] \circ\left(\mathbf{a}_{\iota, \bar{L}, \iota}^{-1} \times \bar{\iota}\right) \circ \\
& {\left[\left(\iota \times \operatorname{coev}_{R}\right) \times \bar{\iota}\right] \circ\left[\left(\iota \times \mathrm{ev}_{L}\right) \times \bar{l}\right] \circ\left(\mathbf{a}_{\iota, \bar{L}, \iota} \times \bar{\iota}\right) \circ\left[\left(x_{i} \times \iota\right) \times \bar{\iota}\right] \circ \mathbf{a}_{\iota \times \bar{l}, \iota, \bar{\iota}}^{-1} \circ} \\
& {\left[(\iota \times \bar{\iota}) \times \operatorname{coev}_{L}\right] \circ \mathbf{r}_{\iota \times \bar{\iota}}^{-1}=a .}
\end{aligned}
$$

Analogously,
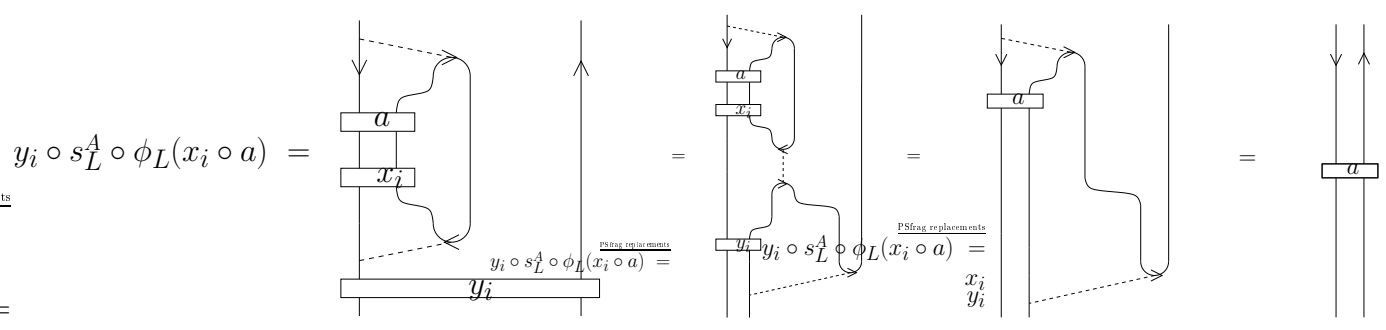

The following lemma collects some useful properties of the quasi-basis $y_{i} \otimes x_{i}$ :

Lemma 3.3 For any elements $a, a_{1}, a_{2}, a_{3}$ in $A$ the following hold true:

$$
\begin{array}{cc}
\text { i) } & a \circ y_{i} \otimes x_{i}=y_{i} \otimes x_{i} \circ a \quad \text { as elements of } A^{L} \otimes{ }_{L} A \\
\text { ii) } & y_{i} \otimes x_{i} * a=y_{i} * S_{A}(a) \otimes x_{i} \quad \text { as elements of } A^{L} \otimes{ }_{L} A \\
\text { iii) } & a_{1} *\left(a_{2} \circ a_{3}\right)=\left[\left(a_{1} \circ y_{i}\right) * a_{2}\right] \circ\left(x_{i} * a_{3}\right) \quad \text { as elements of } A .
\end{array}
$$

Proof: The part $i$ ) is a standard consequence of Proposition 3.2

In order to prove the part $i i)$ use the identity $\phi_{L}\left(a_{1} \circ\left(a_{2} * S_{A}\left(a_{3}\right)\right)\right)=\phi_{L}\left(\left(a_{1} * a_{3}\right) \circ a_{2}\right)$ - following from (3.2) and the coherence axioms in $\mathcal{C}$ - holding true for any $a_{1}, a_{2}, a_{3} \in A$ and Proposition 3.2 to show that in $A^{L} \otimes{ }_{L} A$ we have

$$
\begin{aligned}
y_{i} \otimes x_{i} * a & =y_{i} \otimes s_{L}^{A} \circ \phi_{L}\left(\left(x_{i} * a\right) \circ y_{j}\right) \circ x_{j}=y_{i} \circ s_{L}^{A} \circ \phi_{L}\left(\left(x_{i} * a\right) \circ y_{j}\right) \otimes x_{j}= \\
& =y_{i} \circ s_{L}^{A} \circ \phi_{L}\left(x_{i} \circ\left(y_{j} * S_{A}(a)\right)\right) \otimes x_{j}=y_{j} * S_{A}(a) \otimes x_{j} .
\end{aligned}
$$

The part iii) is checked by direct calculation making use of the definition (3.3), the quasi-basis property (3.14) and the coherence axioms in $\mathcal{C}$.

We are ready to construct the various ingredients of the Hopf algebroid structure on $A$. In addition to the map $s_{L}^{A}$ in (3.17) - that is going to be the source map of the left bialgebroid structure - with the help of the map $\mu$ in (3.9) introduce the map that is going to be the target map as

$$
t_{L}^{A}: L^{o p} \rightarrow A \quad l \mapsto \iota \times \mu(l) .
$$


It is obviously a ring homomorphism. The relations (3.2) imply that the element $i_{A}=\operatorname{coev}_{L} \circ \mathrm{ev}_{R} \in A$ satisfies

$$
s_{L}^{A} \circ \phi_{L}\left(a \circ i_{A}\right) \circ i_{A}=a \circ i_{A}=t_{L}^{A} \circ \phi_{L}\left(a \circ i_{A}\right) \circ i_{A}
$$

for any element $a$ in $A$ and for the map $\phi_{L}$ introduced in (3.18).

Defining the ring homomorphisms that are to be the source and target maps of the right bialgebroid structure on $A$ as

$$
\begin{array}{rll}
s_{R}^{A}: & R \rightarrow A & r \mapsto \iota \times r \\
t_{R}^{A}: \quad R^{o p} \rightarrow A & r \mapsto \nu^{-1}(r) \times \bar{\iota}
\end{array}
$$

and the maps that are going to be the counits as

$$
\begin{aligned}
\pi_{L}^{A}: A \rightarrow L & & a \mapsto \phi_{L}\left(a \circ i_{A}\right) \\
\pi_{R}^{A}: A \rightarrow R & & a \mapsto \nu \circ \phi_{L}\left(S_{A}^{-1}(a) \circ i_{A}\right)
\end{aligned}
$$

we can prove

Lemma 3.4 The maps $\pi_{L}^{A}$ and $\pi_{R}^{A}$ together with the maps (3.17), (3.22) and (3.24.3.25) satisfy

$$
\begin{aligned}
\text { i) } & \left(a \circ i_{A}\right) * 1_{A}=s_{L}^{A} \circ \pi_{L}^{A}(a) \\
\text { ii) } & 1_{A} *\left(a \circ i_{A}\right)=t_{L}^{A} \circ \pi_{L}^{A}(a) \\
\text { iii) } & 1_{A} *\left(i_{A} \circ a\right)=s_{R}^{A} \circ \pi_{R}^{A}(a) \\
\text { iv) } & \left(i_{A} \circ a\right) * 1_{A}=t_{R}^{A} \circ \pi_{R}^{A}(a)
\end{aligned}
$$

for all $a \in A$.

Proof: Using (3.18) and (3.2) one computes that

$$
\begin{aligned}
\pi_{L}^{A}(a) & =\phi_{L}\left(a \circ i_{A}\right)=\mathbf{r}_{\iota} \circ\left(\iota \times \mathrm{ev}_{L}\right) \circ \mathbf{a}_{\iota, \bar{L}, \iota} \circ\left[\left(a \circ \operatorname{coev}_{L} \circ \mathrm{ev}_{R}\right) \times \iota\right] \circ \mathbf{a}_{\iota, \bar{L}, \iota}^{-1} \circ\left(\iota \times \operatorname{coev}_{R}\right) \circ \mathbf{r}_{\iota}^{-1}= \\
& =\mathbf{r}_{\iota} \circ\left(\iota \times \operatorname{ev}_{L}\right) \circ \mathbf{a}_{\iota, \bar{c}, \iota} \circ\left[\left(a \circ \operatorname{coev}_{L}\right) \times \iota\right] \circ \mathbf{l}_{\iota}^{-1}
\end{aligned}
$$

Then $i$ ) follows by

$$
\begin{aligned}
\left(a \circ i_{A}\right) * 1_{A}= & \left(\mathbf{r}_{\iota} \times \bar{\iota}\right) \circ\left[\left(\iota \times \mathrm{ev}_{L}\right) \times \bar{\iota}\right] \circ\left(\mathbf{a}_{\iota, \bar{\iota}, \iota} \times \bar{\iota}\right) \circ \mathbf{a}_{\iota \times \bar{l}, \iota, \bar{\iota}}^{-1} \circ\left[\left(a \circ \operatorname{coev}_{L} \circ \operatorname{ev}_{R}\right) \times(\iota \times \bar{\iota})\right] \circ \mathbf{a}_{\iota \times \bar{l}, \iota, \bar{\iota}} \circ \\
& \left(\mathbf{a}_{\iota, \bar{\iota}, \iota}^{-1} \times \bar{\iota}\right) \circ\left[\left(\iota \times \operatorname{coev}_{R}\right) \times \bar{\iota}\right] \circ\left(\mathbf{r}_{\iota}^{-1} \times \bar{\iota}\right)= \\
= & \left(\mathbf{r}_{\iota} \times \bar{\iota}\right) \circ\left[\left(\iota \times \mathrm{ev}_{L}\right) \times \bar{\iota}\right] \circ\left(\mathbf{a}_{\iota, \bar{\iota}, \iota} \times \bar{\iota}\right) \circ\left[\left(\left(a \circ \operatorname{coev}_{L} \circ \operatorname{ev}_{R}\right) \times \iota\right) \times \bar{\iota}\right] \circ\left(\mathbf{a}_{\iota, \bar{\iota}, \iota}^{-1} \times \bar{\iota}\right) \circ \\
& {\left[\left(\iota \times \operatorname{coev}_{R}\right) \times \bar{\iota}\right] \circ\left(\mathbf{r}_{\iota}^{-1} \times \bar{\iota}\right)=} \\
= & \left(\mathbf{r}_{\iota} \times \bar{\iota}\right) \circ\left[\left(\iota \times \operatorname{ev}_{L}\right) \times \bar{\iota}\right] \circ\left(\mathbf{a}_{\iota, \bar{\iota}, \iota} \times \bar{\iota}\right) \circ\left[\left(\left(a \circ \operatorname{coev}_{L}\right) \times \iota\right) \times \bar{\iota}\right] \circ\left(\mathbf{l}_{\iota}^{-1} \times \bar{\iota}\right)= \\
= & \pi_{L}^{A}(a) \times \bar{\iota}=s_{L}^{A} \circ \pi_{L}^{A}(a) .
\end{aligned}
$$

Analogously, ii) follows by 


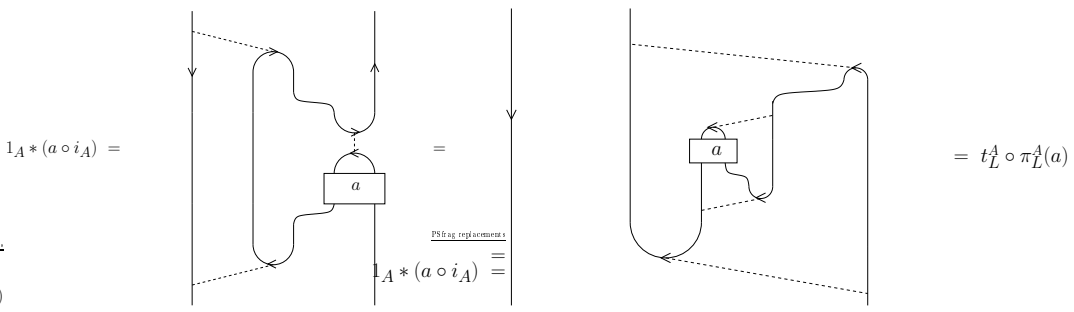

The identities $i i i)$ and $i v$ ) are proven analogously using

$$
\pi_{R}^{A}(a)=\mathbf{r}_{\bar{\iota}} \circ\left(\bar{\iota} \times \operatorname{ev}_{R}\right) \circ(\bar{\iota} \times a) \circ \mathbf{a}_{\bar{\iota}, \iota, \bar{\iota}} \circ\left(\operatorname{coev}_{R} \times \bar{\iota}\right) \circ \mathbf{l}_{\bar{\iota}}^{-1}
$$

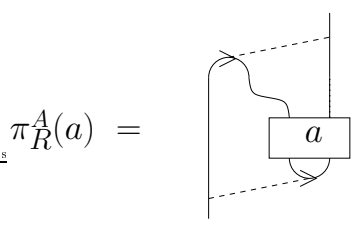

In the following theorem we give the explicit form of the bialgebroid structures on the ring $A$ the existence of which follows by Theorem 3.5 in 25$]$ :

Theorem 3.5 The ring $A$ carries a left bialgebroid structure $\mathcal{A}_{L}$ over the base L and a right bialgebroid structure $\mathcal{A}_{R}$ over $R$ with the structural maps

$$
\begin{array}{ll}
s_{L}^{A}(l)=l \times \bar{\iota} & t_{L}^{A}(l)=\iota \times \mu(l) \\
\gamma_{L}^{A}(a)=a * S_{A}^{-1}\left(y_{i}\right) \otimes x_{i}=S_{A}^{-1}\left(y_{i}\right) \otimes x_{i} * a & \pi_{L}^{A}(a)=\phi_{L}\left(a \circ i_{A}\right) \\
s_{R}^{A}(r)=\iota \times r & t_{R}^{A}(r)=\nu^{-1}(r) \times \bar{\iota} \\
\gamma_{R}^{A}(a)=a * S_{A}\left(x_{i}\right) \otimes y_{i}=S_{A}\left(x_{i}\right) \otimes y_{i} * a & \pi_{R}^{A}(a)=\nu \circ \phi_{L}\left(S_{A}^{-1}(a) \circ i_{A}\right) .
\end{array}
$$

Proof: The equality of the two forms of $\gamma_{L}^{A}$ given in (3.29) follows from (3.20) and the fact that by (3.7) we have $S_{A}\left(a_{1} * a_{2}\right)=S_{A}\left(a_{2}\right) * S_{A}\left(a_{1}\right)$.

Since

$$
\left(s_{L}^{A}(l) \circ a_{1}\right) * a_{2}=s_{L}^{A}(l) \circ\left(a_{1} * a_{2}\right) \quad \text { and } \quad a_{1} *\left(t_{L}^{A}(l) \circ a_{2}\right)=t_{L}^{A}(l) \circ\left(a_{1} * a_{2}\right)
$$

the $\gamma_{L}^{A}$ is an $L$ - $L$ bimodule map. The left $L$-module map property of $\pi_{L}^{A}$ follows from the left $L$-module property of $\phi_{L}$. Its right $L$-module map property follows by the use of the identity $\operatorname{ev}_{L} \circ(\mu(l) \times \iota)=$ $\mathrm{ev}_{L} \circ(\bar{\iota} \times l)$ and the relations (3.2):

$$
\begin{aligned}
\pi_{L}^{A}\left(t_{L}^{A}(l) \circ a\right) & =\phi_{L}\left(t_{L}^{A}(l) \circ a \circ i_{A}\right)= \\
& =\mathbf{r}_{\iota} \circ\left(\iota \times \mathrm{ev}_{L}\right) \circ \mathbf{a}_{\iota, \bar{\iota}, \iota} \circ[(\iota \times \bar{\iota}) \times l] \circ(a \times \iota) \circ\left(\operatorname{coev}_{L} \times \iota\right) \circ \mathbf{l}_{\iota}^{-1}= \\
& =\mathbf{r}_{\iota} \circ\left(\iota \times \operatorname{ev}_{L}\right) \circ \mathbf{a}_{\iota, \bar{\iota}, \iota} \circ(a \times \iota) \circ\left(\operatorname{coev}_{L} \times \iota\right) \circ \mathbf{l}_{\iota}^{-1} \circ l=\pi_{L}^{A}(a) \circ l .
\end{aligned}
$$

The coassociativity of $\gamma_{L}^{A}$ follows by using both forms of it:

$$
\left(\gamma_{L}^{A} \otimes \operatorname{id}_{A}\right) \circ \gamma_{L}^{A}(a)=S_{A}^{-1}\left(y_{j}\right) \otimes x_{j} * a * S_{A}^{-1}\left(y_{i}\right) \otimes x_{i}=\left(\operatorname{id}_{A} \otimes \gamma_{L}^{A}\right) \circ \gamma_{L}^{A}(a)
$$

The relations (3.113.12) imply that $S_{A} \circ t_{L}^{A}(l)=s_{L}^{A}(l)$. Since by Proposition 3.2 and (3.23) we have $y_{i} \circ s_{L}^{A} \circ \pi_{L}^{A}\left(x_{i}\right)=i_{A}=s_{L}^{A} \circ \pi_{L}^{A} \circ S_{A}^{-1}\left(y_{i}\right) \circ x_{i}$ and $i_{A}$ is the unit for $\hat{A}$, the $\pi_{L}^{A}$ is the counit for $\gamma_{L}^{A}$.

Using (3.19) we have the identity in $A_{L} \otimes{ }_{L} A$ for any $a \in A$ and $l \in L$ :

$$
\begin{aligned}
a_{(1)} \circ t_{L}^{A}(l) \otimes a_{(2)} & =S_{A}^{-1}\left(y_{i}\right) \circ t_{L}^{A}(l) \otimes x_{i} * a=S_{A}^{-1}\left(y_{i}\right) \otimes\left(x_{i} \circ s_{L}^{A}(l)\right) * a= \\
& =S_{A}^{-1}\left(y_{i}\right) \otimes\left(x_{i} * a\right) \circ s_{L}^{A}(l)=a_{(1)} \otimes a_{(2)} \circ s_{L}^{A}(l) .
\end{aligned}
$$


Since $a * 1_{A}=s_{L}^{A} \circ \phi_{L}(a)$ for all $a \in A$, also

$$
\gamma_{L}^{A}\left(1_{A}\right)=S_{A}^{-1}\left(y_{i}\right) \otimes x_{i} * 1_{A}=S_{A}^{-1}\left(y_{i}\right) \otimes s_{L}^{A} \circ \phi_{L}\left(x_{i}\right)=t_{L}^{A} \circ \phi_{L}\left(x_{i}\right) \circ S_{A}^{-1}\left(y_{i}\right) \otimes 1_{A}=1_{A} \otimes 1_{A} .
$$

It follows from (3.21) that

$$
\begin{aligned}
\gamma_{L}^{A}\left(a_{1}\right) \gamma_{L}^{A}\left(a_{2}\right) & =S_{A}^{-1}\left(y_{i}\right) \circ S_{A}^{-1}\left(y_{j}\right) \otimes\left(x_{i} * a_{1}\right) \circ\left(x_{j} * a_{2}\right)= \\
& =S_{A}^{-1}\left(y_{k} \circ s_{L}^{A} \circ \phi_{L}\left(x_{k} \circ y_{j} \circ y_{i}\right)\right) \otimes\left(x_{i} * a_{1}\right) \circ\left(x_{j} * a_{2}\right)= \\
& =S_{A}^{-1}\left(y_{k}\right) \otimes s_{L}^{A} \circ \phi_{L}\left(x_{k} \circ y_{j} \circ y_{i}\right) \circ\left(x_{i} * a_{1}\right) \circ\left(x_{j} * a_{2}\right)= \\
& =S_{A}^{-1}\left(y_{k}\right) \otimes\left[\left(x_{k} \circ y_{j}\right) * a_{1}\right] \circ\left(x_{j} * a_{2}\right)=S_{A}^{-1}\left(y_{k}\right) \otimes x_{k} *\left(a_{1} \circ a_{2}\right)=\gamma_{L}^{A}\left(a_{1} \circ a_{2}\right) .
\end{aligned}
$$

Finally, using (3.23) we have

$\pi_{L}^{A}\left(a_{1} \circ s_{L}^{A} \circ \pi_{L}^{A}\left(a_{2}\right)\right)=\phi_{L}\left(a_{1} \circ s_{L}^{A} \circ \phi_{L}\left(a_{2} \circ i_{A}\right) \circ i_{A}\right)=\phi_{L}\left(a_{1} \circ a_{2} \circ i_{A}\right)=\pi_{L}^{A}\left(a_{1} \circ a_{2}\right)=\pi_{L}^{A}\left(a_{1} \circ t_{L}^{A} \circ \pi_{L}^{A}\left(a_{2}\right)\right)$.

This finishes the proof of the statement that $\mathcal{A}_{L}=\left(A, L, s_{L}^{A}, t_{L}^{A}, \gamma_{L}^{A}, \pi_{L}^{A}\right)$ is a left bialgebroid.

In order to prove that $\mathcal{A}_{R}=\left(A, R, s_{R}^{A}, t_{R}^{A}, \gamma_{R}^{A}, \pi_{R}^{A}\right)$ is a right bialgebroid observe that $\left(S_{A}, \nu\right)$ is a left bialgebroid isomorphism $\mathcal{A}_{L} \rightarrow\left(\mathcal{A}_{R}\right)_{\text {cop }}^{o p}$ i. e.

$$
\begin{array}{ll}
S_{A} \circ s_{L}^{A}=s_{R}^{A} \circ \nu & S_{A} \circ t_{L}^{A}=t_{R}^{A} \circ \nu \\
S_{A \otimes_{L} A} \circ \gamma_{L}^{A}=\gamma_{R}^{A} \circ S_{A} & \nu \circ \pi_{L}^{A}=\pi_{R}^{A} \circ S_{A}
\end{array}
$$

where the map $S_{A \otimes_{L} A}: A_{L} \otimes{ }_{L} A \rightarrow A^{R} \otimes{ }^{R} A$ is defined as $a_{1} \otimes a_{2} \mapsto S_{A}\left(a_{2}\right) \otimes S_{A}\left(a_{1}\right)$ and $\nu: L \rightarrow R^{o p}$ has been introduced in (3.9).

Theorem 3.6 The left and right bialgebroid structures (3.29) on the ring $A$ and the map $S_{A}$ in (3.8) form a Hopf algebroid $\mathcal{A}$.

Proof: It is obvious from (3.17), (3.22) and (3.243.25) that

$$
s_{L}^{A}(L)=t_{R}^{A}(R) \quad t_{L}^{A}(L)=s_{R}^{A}(R)
$$

as subrings of $A$.

Using the explicit forms (3.29) of the coproducts $\gamma_{L}^{A}$ and $\gamma_{R}^{A}$ we have

$$
\begin{aligned}
& \left(\gamma_{L}^{A} \otimes \operatorname{id}_{A}\right) \circ \gamma_{R}^{A}(a)=S_{A}^{-1}\left(y_{j}\right) \otimes x_{j} * a * S_{A}\left(x_{i}\right) \otimes y_{i}=\left(\operatorname{id}_{A} \otimes \gamma_{R}^{A}\right) \circ \gamma_{L}^{A}(a) \\
& \left(\gamma_{R}^{A} \otimes \operatorname{id}_{A}\right) \circ \gamma_{L}^{A}(a)=S_{A}\left(x_{j}\right) \otimes y_{j} * a * S_{A}^{-1}\left(y_{i}\right) \otimes x_{i}=\left(\operatorname{id}_{A} \otimes \gamma_{L}^{A}\right) \circ \gamma_{R}^{A}(a) .
\end{aligned}
$$

The map $S_{A}$ is bijective by the bijectivity of the maps $\mathcal{F}$ and $\dot{\mathcal{F}}$. It is anti-multiplicative by (3.7) and (3.113.12) imply $S_{A} \circ t_{L}^{A}=s_{L}^{A}$ and $S_{A} \circ t_{R}^{A}=s_{R}^{A}$. Hence

$$
S_{A}\left(t_{L}^{A}(l) \circ a \circ t_{L}^{A}\left(l^{\prime}\right)\right)=s_{L}^{A}\left(l^{\prime}\right) \circ S_{A}(a) \circ s_{L}^{A}(l) \quad S_{A}\left(t_{R}^{A}(r) \circ a \circ t_{R}^{A}\left(r^{\prime}\right)\right)=s_{R}^{A}\left(r^{\prime}\right) \circ S_{A}(a) \circ s_{R}^{A}(r) .
$$

By (3.21), the iii) and the ii) of Lemma 3.4 also

$$
\begin{aligned}
& S_{A}\left(a_{(1)}\right) \circ a_{(2)}=y_{i} \circ\left(x_{i} * a\right)=1_{A} *\left(i_{A} \circ a\right)=s_{R}^{A} \circ \pi_{R}^{A}(a) \\
& a^{(1)} \circ S_{A}\left(a^{(2)}\right)=S_{A}\left(\left(y_{i} * a\right) \circ x_{i}\right)=S_{A}\left(1_{A} *\left(a \circ i_{A}\right)\right)=S_{A} \circ t_{L}^{A} \circ \pi_{L}^{A}(a)=s_{L}^{A} \circ \pi_{L}^{A}(a) .
\end{aligned}
$$

Theorem 3.6] above generalizes the result proven in the Proposition 6.19 in [14. There $\iota$ is assumed to be a finite index and irreducible D2 Frobenius 1-morphism in a semisimple $k$-linear bicategory. Then the endomorphism rings $A$ of $\iota \times \bar{\iota}$ and $B$ of $\bar{\iota} \times \iota$ are equipped with dual finite dimensional semisimple and cosemisimple Hopf algebra structures.

Interchanging the roles of the 1-morphisms $\iota$ and $\bar{\iota}$, Theorem 3.6 implies that also the ring $B$ carries a Hopf algebroid structure $\mathcal{B}=\left(\mathcal{B}_{L}, \mathcal{B}_{R}, S_{B}\right)$. The left bialgebroid $\mathcal{B}_{L}$ has $R$ as the base and the structural maps

$$
\begin{aligned}
& s_{L}^{B}(r)=r \times \iota \quad t_{L}^{B}(r)=\bar{\iota} \times \nu^{-1}(r) \\
& \gamma_{L}^{B}(b)=b * \mathcal{F}\left(x_{i}\right) \otimes \mathcal{F}\left(y_{i}\right) \equiv \mathcal{F}\left(x_{i}\right) \otimes \mathcal{F}\left(y_{i}\right) * b \quad \pi_{L}^{B}(b)=\nu \circ \phi_{L} \circ \mathcal{F}^{-1}(b) .
\end{aligned}
$$


The right bialgebroid $\mathcal{B}_{R}$ has $L$ as the base and the structural maps

$$
\begin{array}{ll}
s_{R}^{B}(l)=\bar{\imath} \times l & t_{R}^{B}(l)=\mu(l) \times \iota \\
\gamma_{R}^{B}(b)=b * \dot{\mathcal{F}}\left(y_{i}\right) \otimes \dot{\mathcal{F}}\left(x_{i}\right) \equiv \dot{\mathcal{F}}\left(y_{i}\right) \otimes \dot{\mathcal{F}}\left(x_{i}\right) * b & \pi_{R}^{B}(b)=\phi_{L} \circ \dot{\mathcal{F}}^{-1}(b) .
\end{array}
$$

In the rest of this subsection we are going to prove that the Hopf algebroid $\mathcal{B}$ is strictly isomorphic to the dual of $\mathcal{A}$.

Lemma 3.7 The four rings ${ }_{*} \mathcal{A}$ and $\mathcal{A}_{*}$ in (2.10), $\mathcal{A}^{*}$ and ${ }^{*} \mathcal{A}$ in (2.11) are all isomorphic to $B$.

Proof: We construct the isomorphism

$$
\alpha^{*}: B \rightarrow \mathcal{A}^{*} \quad b \mapsto \nu \circ \phi_{L}\left(S_{A}^{-1}(-) \circ \mathcal{F}^{-1}(b)\right)
$$

with inverse

$$
\alpha^{*-1}: \mathcal{A}^{*} \rightarrow B \quad \phi^{*} \mapsto \mathcal{F}\left(y_{i} \circ t_{R}^{A} \circ \phi^{*} \circ S_{A}\left(x_{i}\right)\right)
$$

As a matter of fact

$$
\begin{aligned}
\alpha^{*-1} \circ \alpha^{*}(b) & =\mathcal{F}\left(y_{i} \circ t_{R}^{A} \circ \nu \circ \phi_{L}\left(x_{i} \circ \mathcal{F}^{-1}(b)\right)\right)=b \\
\alpha^{*} \circ \alpha^{*-1}\left(\phi^{*}\right) & =\nu \circ \phi_{L}\left(S_{A}^{-1}(-) \circ y_{i} \circ t_{R}^{A} \circ \phi^{*} \circ S_{A}\left(x_{i}\right)\right)=\nu \circ \phi_{L}\left(S_{A}^{-1}(-) \circ y_{i}\right) \circ \phi^{*} \circ S_{A}\left(x_{i}\right)= \\
& =\phi^{*} \circ S_{A}\left(s_{L}^{A} \circ \phi_{L}\left(S_{A}^{-1}(-) \circ y_{i}\right) \circ x_{i}\right)=\phi^{*} \\
\left(\alpha^{*}\left(b_{1}\right) \alpha^{*}\left(b_{2}\right)\right)(a) & =\nu \circ \phi_{L}\left(S_{A}^{-1}\left[\left(y_{i} * a\right) \circ t_{R}^{A} \circ \nu \circ \phi_{L}\left(x_{i} \circ \mathcal{F}^{-1}\left(b_{2}\right)\right)\right] \circ \mathcal{F}^{-1}\left(b_{1}\right)\right)= \\
& =\nu \circ \phi_{L}\left(\left[S_{A}^{-1}(a) * S_{A}^{-1} \circ \mathcal{F}^{-1}\left(b_{2}\right)\right] \circ \mathcal{F}^{-1}\left(b_{1}\right)\right)= \\
& \left.=\nu \circ \phi_{L}\left(S_{A}^{-1}(a) \circ\left[\mathcal{F}^{-1}\left(b_{1}\right) * \mathcal{F}^{-1}\left(b_{2}\right)\right]\right)=\nu \circ \phi_{L}\left(S_{A}^{-1}(a) \circ \mathcal{F}^{-1}\left(b_{1} \circ b_{2}\right)\right]\right)= \\
& =\alpha^{*}\left(b_{1} \circ b_{2}\right)(a) .
\end{aligned}
$$

Analogously, one checks that

$$
\begin{aligned}
& { }^{*} \alpha: \quad B \rightarrow{ }^{*} \mathcal{A} \quad b \quad \mapsto \quad \nu \circ \phi_{L}\left(\mathcal{F}^{-1}(b) \circ-\right) \\
& { }^{*} \alpha^{-1}:{ }^{*} \mathcal{A} \rightarrow B \quad{ }^{*} \phi \quad \mapsto \mathcal{F}\left(t_{R}^{A} \circ{ }^{*} \phi\left(y_{i}\right) \circ x_{i}\right) \\
& { }_{*} \alpha: \quad B \rightarrow{ }_{*} \mathcal{A} \quad b \mapsto \phi_{L}\left(-\circ \dot{\mathcal{F}}^{-1}(b)\right. \\
& { }_{*} \alpha^{-1}:{ }_{*} \mathcal{A} \rightarrow B \quad{ }_{*} \phi \quad \mapsto \dot{\mathcal{F}}\left(y_{i} \circ s_{L}^{A} \circ{ }_{*} \phi\left(x_{i}\right)\right) \\
& \alpha_{*}: \quad B \quad \rightarrow \quad \mathcal{A}_{*} \quad b \quad \mapsto \phi_{L}\left(\dot{\mathcal{F}}^{-1}(b) \circ S_{A}(-)\right) \\
& \alpha_{*}{ }^{-1}: \mathcal{A}_{*} \rightarrow B \quad \phi_{*} \mapsto \dot{\mathcal{F}}\left(s_{L}^{A} \circ \phi_{*} \circ S_{A}^{-1}\left(y_{i}\right) \circ x_{i}\right)
\end{aligned}
$$

define isomorphisms of rings.

Theorem 3.8 The element $i_{A}:=\operatorname{coev}_{L} \circ \mathrm{ev}_{R}$ is a two sided non-degenerate integral in the Hopf algebroid $\mathcal{A}$.

Proof: The element $i_{A}$ is a left integral by (3.23) and the definition (3.27) of the map $\pi_{L}^{A}$. Since it is invariant under $S_{A}$ it is also a right integral. It remains to check non-degeneracy. As a matter of fact

$$
\alpha^{*}(b) \rightarrow i_{A}=y_{i} \circ t_{R}^{A} \circ \nu \circ \phi_{L}\left(x_{i} \circ \mathcal{F}^{-1}(b)\right)=\mathcal{F}^{-1}(b)
$$

hence the map

$$
\left(i_{A}\right)_{R}=\mathcal{F}^{-1} \circ \alpha^{*-1}
$$

is bijective. Analogously, ${ }_{R}\left(i_{A}\right)=\dot{\mathcal{F}}^{-1} \circ{ }^{*} \alpha^{-1}$ is bijective.

Interchanging the roles of the 1-morphisms $\iota$ and $\bar{\iota}$, Theorem 3.8 implies that $i_{B}=\operatorname{coev}_{R} \circ \operatorname{ev}_{L}$ is a two sided non-degenerate integral in the Hopf algebroid $\mathcal{B}$.

Remark 3.9 With the help of the two sided non-degenerate integral $i_{A}=\operatorname{coev}_{L} \circ \mathrm{ev}_{R}$ the convolution product (3.3) takes the forms

$$
\begin{aligned}
a_{1} * a_{2} & =\left(i_{A}\right)_{R}\left(\left(i_{A}\right)_{R}^{-1}\left(a_{1}\right)\left(i_{A}\right)_{R}^{-1}\left(a_{2}\right)\right) \equiv{ }_{R}\left(i_{A}\right)\left({ }_{R}\left(i_{A}\right)^{-1}\left(a_{2}\right)_{R}\left(i_{A}\right)^{-1}\left(a_{1}\right)\right) \equiv \\
& =\left(i_{A}\right)_{L}\left(\left(i_{A}\right)_{L}^{-1}\left(a_{2}\right)\left(i_{A}\right)_{L}^{-1}\left(a_{1}\right)\right) \equiv_{L}\left(i_{A}\right)\left({ }_{L}\left(i_{A}\right)^{-1}\left(a_{1}\right)_{L}\left(i_{A}\right)^{-1}\left(a_{2}\right)\right)
\end{aligned}
$$

where $i_{R}: A^{R} \rightarrow A,{ }_{R} i:{ }^{R} A \rightarrow A,{ }_{L} i:{ }_{L} A \rightarrow A$ and $i_{L}: A_{L} \rightarrow A$ are bijections of additive groups. 
By Theorems 3.8 and 2.8 there exists a Hopf algebroid structure $\mathcal{A}^{*}{ }_{i_{A}}$ on the ring $\mathcal{A}^{*}$ with structural maps listed in the Theorem 2.8

Theorem 3.10 The Hopf algebroid $\mathcal{A}^{*}{ }_{i_{A}}$ is strictly isomorphic to $\mathcal{B}$.

Proof: Let $\alpha^{*}: B \rightarrow \mathcal{A}^{*}$ be the ring isomorphism (3.33). We claim that $\left(\alpha^{*}, \mathrm{id}_{R}\right)$ is a strict isomorphism of Hopf algebroids $\mathcal{B} \rightarrow \mathcal{A}^{*} i_{A}$. Use (3.11) and (3.35) to check that

$$
\begin{aligned}
\alpha^{*-1} \circ s_{L}^{*}(r) & \left.=\mathcal{F}\left(y_{i} \circ t_{R}^{A}\left[r \circ \pi_{R}^{A} \circ S_{A}\left(x_{i}\right)\right]\right)=\mathcal{F}\left(y_{i} \circ s_{L}^{A} \circ \pi_{L}^{A}\left(x_{i}\right) \circ t_{R}^{A}(r)\right]\right)= \\
& =\mathcal{F}\left(i_{A} \circ t_{R}^{A}(r)\right)=r \times \iota=s_{L}^{B}(r) \\
\alpha^{*-1} \circ t_{L}^{*}(r) & =\mathcal{F}\left(y_{i} \circ t_{R}^{A} \circ \pi_{R}^{A}\left(s_{R}^{A}(r) \circ S_{A}\left(x_{i}\right)\right)\right)=\mathcal{F}\left(t_{R}^{A}(r) \circ y_{i} \circ s_{L}^{A} \circ \pi_{L}^{A}\left(x_{i}\right)\right)= \\
& =\mathcal{F}\left(t_{R}^{A}(r) \circ i_{A}\right)=\bar{\iota} \times \nu^{-1}(r)=t_{L}^{B}(r) \\
\left(\alpha^{*-1} \otimes \alpha^{*-1}\right) \circ \gamma_{L}^{*} \circ \alpha^{*}(b) & =\alpha^{*-1}\left(\alpha^{*}(b) \leftarrow S_{A}\left(x_{i}\right)\right) \otimes \alpha^{*-1} \circ\left(i_{A}\right)_{R}^{-1}\left(y_{i}\right)= \\
& =\mathcal{F}\left(x_{i} \circ y_{j} \circ t_{R}^{A} \circ \nu \circ \phi_{L}\left(x_{j} \circ \mathcal{F}^{-1}(b)\right)\right) \otimes \mathcal{F}\left(y_{i}\right)= \\
& =\mathcal{F}\left(x_{i} \circ \mathcal{F}^{-1}(b)\right) \otimes \mathcal{F}\left(y_{i}\right)=\gamma_{L}^{B}(b) \\
\pi_{L}^{*} \circ \alpha^{*}(b) & =\alpha^{*}(b)\left(1_{A}\right)=\nu \circ \phi_{L} \circ \mathcal{F}^{-1}(b)=\pi_{L}^{B}(b) \\
\alpha^{*-1} \circ S^{*} \circ \alpha^{*} & =\alpha^{*-1} \circ\left(i_{A}\right)_{R}^{-1} \circ S_{A} \circ\left(i_{A}\right)_{R} \circ \alpha^{*}=\mathcal{F} \circ S_{A} \circ \mathcal{F}^{-1}=\mathcal{F} \circ \dot{\mathcal{F}}^{-1}=S_{B} .
\end{aligned}
$$

Example 3.11 Let $N \rightarrow M$ be a D2 Frobenius extension of rings. Then the $N-M$ bimodule ${ }_{N} M_{M}$ is a D2 Frobenius 1-morphism in the additive bicategory of bimodules. Applying the above construction we obtain the Hopf algebroid described in Section 3 of [2].

\subsection{The inverse construction}

In this subsection we address the question what Hopf algebroids arise as symmetries of abstract D2 Frobenius extensions in the way explaned in Subsection 3.2 By Theorem 3.8 the existence of a two sided non-degenerate integral is a necessary condition. The main result of this subsection states that it is also sufficient.

Throughout this subsection let $\mathcal{H}=\left(\mathcal{H}_{L}, \mathcal{H}_{R}, S\right)$ be a Hopf algebroid with a non-degenerate right integral $i$. We use the notation $\mathcal{H}_{L}=\left(H, L, s_{L}, t_{L}, \gamma_{L}, \pi_{L}\right)$ and $\mathcal{H}_{R}=\left(H, R, s_{R}, t_{R}, \gamma_{R}, \pi_{R}\right)$. In what follows we associate a bicategory and a D2 Frobenius 1-morphism of it to the pair $(\mathcal{H}, i)$. The construction is built on the generalization of the result in [31] described in the Appendix. We arrive to the statement that if $i$ is a two sided non-degenerate integral then the Hopf algebroid symmetry of the D2 Frobenius 1-morphism constructed is isomorphic to $\mathcal{H}$.

Recall that for a right bialgebroid $\mathcal{H}_{R}$ the right regular $H$-module $H_{H}$ is the object part of a comonoid. That is the triple $\left(H_{H}, \gamma_{R}, \pi_{R}\right)$ is a comonoid in the monoidal category $\mathcal{M}_{H}$. Now we claim that for a Hopf algebroid $\mathcal{H}$ possessing a non-degenerate right integral the $H_{H}$ has more structure then just being a comonoid. The following proposition generalizes the result of [14] on Hopf algebras:

Proposition 3.12 Let $\mathcal{H}$ be a Hopf algebroid with a non-derenerate right integral $i$. Define the convolution product on the additive group $\mathrm{H}$ underlying the ring $H$ as

$$
h_{1} * h_{2}={ }_{L} i\left({ }_{L} i^{-1}\left(h_{1}\right){ }_{L} i^{-1}\left(h_{2}\right)\right)
$$

and the map

$$
\eta: R \rightarrow H \quad r \mapsto i s_{R}(r) \equiv i t_{R}(r) .
$$

Then $\left(H_{H}, *, \eta, \gamma_{R}, \pi_{R}\right)$ is a Frobenius algebra in the category $\mathcal{M}_{H}$.

Proof: Let ${ }_{*} \rho:={ }_{L} i^{-1}\left(1_{H}\right)$. We use the notation $\gamma_{L}(h)=h_{(1)} \otimes h_{(2)}$ and $\gamma_{R}(h)=h^{(1)} \otimes h^{(2)}$ for $h \in H$. Using the Hopf algebroid identity $h-{ }_{L} i^{-1}(k)=k<i_{L}{ }^{-1}(h)$ for all $h, k \in H$ - see [2] Lemma 5.11 - the convolution product (3.37) has the equivalent forms

$$
\begin{aligned}
h * k & =h-{ }_{L} i^{-1}(k) \equiv t_{L} \circ_{*} \rho\left(h_{(2)} S(k)\right) h_{(1)}= \\
& =k \leftarrow i_{L}{ }^{-1}(h) \equiv s_{L}{ }{ }_{*} \rho\left(h S\left(k_{(1)}\right)\right) k_{(2)} .
\end{aligned}
$$


First we show that $\left(H_{H}, *, \eta\right)$ is a monoid in $\mathcal{M}_{H}$. Both $*$ and $\eta$ are $H$-module maps as for all $h, k, k^{\prime} \in H$ and $r \in R$ we have

$$
\begin{aligned}
k h^{(1)} * k^{\prime} h^{(2)} & =t_{L} \circ_{*} \rho\left(k_{(2)} h_{(1)}^{(1)} S\left(k^{\prime} h^{(2)}\right)\right) k_{(1)} h_{(1)}^{(1)}=t_{L} \circ_{*} \rho\left(k_{(2)} s_{L} \circ \pi_{L}\left(h_{(2)}\right) S\left(k^{\prime}\right)\right) k_{(1)} h_{(1)}= \\
& =t_{L} \circ{ }_{*} \rho\left(k_{(2)} S\left(k^{\prime}\right)\right) k_{(1)} h=\left(k * k^{\prime}\right) h \\
\eta \circ \pi_{R}\left(s_{R}(r) h\right) & =i s_{R} \circ \pi_{R}\left(s_{R}(r) h\right)=i s_{R}(r) h=\eta(r) h .
\end{aligned}
$$

Using (3.39), the convolution product $*$ can be seen to be associative: for $h, k, l \in H$ we have

$$
(h * k) * l=t_{L} \circ_{*} \rho\left(k_{(3)} S(l)\right) s_{L} \circ_{*} \rho\left(h S\left(k_{(1)}\right)\right) k_{(2)}=h *(k * l) .
$$

As $i$ is the unit for the ring $(\mathrm{H}, *)$, the map $\eta$ is the unit for $*$ :

$$
\begin{aligned}
& h * \eta(r)=h *\left(i s_{R}(r)\right)=(h * i) s_{R}(r)=h s_{R}(r)=r_{H}(h \otimes r) \\
& \eta(r) * h=\left(i t_{R}(r)\right) * h=(i * h) t_{R}(r)=h t_{R}(r)=l_{H}(r \otimes h) .
\end{aligned}
$$

The compatibility of the monoid $\left(H_{H}, *, \eta\right)$ and the comonoid $\left(H_{H}, \gamma_{R}, \pi_{R}\right)$ follows by

$$
\begin{aligned}
\gamma_{R}(h * k) & =\gamma_{R}\left(h-{ }_{L} i^{-1}(k)\right)=h^{(1)} \otimes h^{(2)}-{ }_{L} i^{-1}(k)=h^{(1)} \otimes h^{(2)} * k \\
& =\gamma_{R}\left(k \leftarrow i_{L}{ }^{-1}(h)\right)=k^{(1)} \leftarrow i_{L}{ }^{-1}(h) \otimes k^{(2)}=h * k^{(1)} \otimes k^{(2)} .
\end{aligned}
$$

It is obvious from (3.39) that also

$$
\gamma_{L}(h * k)=h_{(1)} \otimes h_{(2)} * k=h * k_{(1)} \otimes k_{(2)} .
$$

Let $\operatorname{BIM}\left(\mathcal{M}_{H}\right)$ denote the additive bicategory of internal bimodules in the monoidal category $\mathcal{M}_{H}$ of right $H$-modules. (For the definition of the bicategory of internal bimodules see the Appendix.) Denote the trivial monoid corresponding to the monoidal unit $R_{H}$ of $\mathcal{M}_{H}$ by $\mathcal{U}=\left(R_{H}, l_{R}=r_{R}, R\right)$ and the monoid (3.373.38) by $\mathcal{Q}=\left(H_{H}, *, \eta\right)$. Then by Proposition 4.1 the $\mathcal{X}:=\left(H_{H}, l_{H}, *\right)$ is an internal $\mathcal{U}$ - $\mathcal{Q}$ bimodule and $\overline{\mathcal{X}}:=\left(H_{H}, *, r_{H}\right)$ is an internal $\mathcal{Q}-\mathcal{U}$ bimodule in $\mathcal{M}_{H}$. Furthermore, $\overline{\mathcal{X}}$ is the two sided dual of $\mathcal{X}$ in $\operatorname{BIM}\left(\mathcal{M}_{H}\right)$.

Proposition 3.13 The Frobenius 1-morphism $\mathcal{X}$ of the bicategory $\operatorname{BIM}\left(\mathcal{M}_{H}\right)$ satisfies the D2 condition.

Proof: The 1-morphism $\mathcal{X} \underset{\mathscr{Q}}{\otimes} \overline{\mathcal{X}}$ of $\operatorname{BIM}\left(\mathcal{M}_{H}\right)$ is the internal $\mathcal{U}-\mathcal{U}$ bimodule $\left(H_{H}, l_{H}, r_{H}\right)$. Introduce the left multiplication map

$$
\Lambda: H \rightarrow \mathcal{M}_{H}^{1}\left(H_{H}, H_{H}\right) \equiv \operatorname{BIM}\left(\mathcal{M}_{H}\right)^{2}(\mathcal{X} \underset{\mathscr{Q}}{\otimes} \overline{\mathcal{X}}, \mathcal{X} \underset{\mathscr{Q}}{\otimes} \overline{\mathcal{X}}) \quad \Lambda(h) k:=h k .
$$

We construct the D2 quasi-basis

$$
\Lambda\left(S\left(i_{(1)}\right)\right) \otimes \Lambda\left(i_{(2)}\right)
$$

Using the explicit forms of the coherence isomorphisms

$$
\begin{aligned}
\underline{\mathbf{l}}_{\mathcal{X}}(r \otimes h) & =h t_{R}(r) & \underline{\mathbf{l}}-\overline{\mathcal{X}}(h \otimes k) & =h * k \\
\underline{\mathbf{r}}_{\mathcal{X}}(h \otimes k) & =h * k & \underline{\mathbf{X}}(h \otimes r) & =h s_{R}(r) \\
\underline{\mathbf{a}}_{\mathcal{X}, \overline{\mathcal{X}}, \mathcal{X}}(h \otimes k \otimes l) & =h \otimes k \otimes l & \underline{\mathbf{a}}_{\overline{\mathcal{X}}, \mathcal{X}, \overline{\mathcal{X}}}(h \otimes k \otimes l) & =h \otimes k \otimes l
\end{aligned}
$$

for $r \in R, h, k, l \in H$ and the 2-morphisms (4.45) and (4.46) one checks that

$$
\begin{aligned}
\left(\Lambda\left(S\left(i_{(1)}\right)\right) \underset{\mathcal{U}}{\otimes} \mathcal{X}\right) & \circ \gamma_{R} \circ * \circ\left(\Lambda\left(i_{(2)}\right) \otimes \mathcal{U} \mathcal{X}\right)(h \otimes k)= \\
& =S\left(i_{(1)}\right)\left[\left(i_{(2)} h\right) * k\right]^{(1)} \otimes\left[\left(i_{(2)} h\right) * k\right]^{(2)}= \\
& =h S\left(i_{(1)}\right) i_{(2)}(1) \otimes i_{(2)}^{(2)} * k=h s_{R} \circ \pi_{R}\left(i^{(1)}\right) \otimes i^{(2)} * k= \\
& =h \otimes\left(i^{(2)} * k\right) t_{R} \circ \pi_{R}\left(i^{(1)}\right)=h \otimes k
\end{aligned}
$$

which is the D2 quasi-basis property (3.14).

Proposition 3.13 together with Theorem 3.6 implies that the $\operatorname{ring} A:=\operatorname{BIM}\left(\mathcal{M}_{H}\right)^{2}(\mathcal{X} \stackrel{\otimes}{\mathcal{X}}, \mathcal{X} \stackrel{\otimes}{\otimes} \overline{\mathcal{X}})$ carries a Hopf algebriod structure $\mathcal{A}$. 
Theorem 3.14 If the non-degenerate right integral $i$ used to define the convolution product (3.37) is invariant under the antipode then the Hopf algebroid $\mathcal{A}$ is isomorphic to $\mathcal{H}$.

Remark 3.15 The non-degenerate right integral is also a left integral if and only if it is invariant under $S_{A}$. The if part follows from iii) of Lemma 2.7 and the only if part follows by

$$
S_{A}^{-1}(i)=i \leftarrow\left(i \neg{ }_{*} \rho\right)=t_{L} \circ{ }_{*} \rho\left(i_{(2)} i\right) i_{(1)}=t_{L} \circ * \rho\left(s_{L} \circ \pi_{L}\left(i_{(2)}\right) i\right) i_{(1)}=t_{L} \circ * \rho(i) t_{L} \circ \pi_{L}\left(i_{(2)}\right) i_{(1)}=i .
$$

Proof of the Theorem: The total ring of the Hopf algebroid $\mathcal{A}$ is

$$
A:=\operatorname{BIM}\left(\mathcal{M}_{H}\right)^{2}(\mathcal{X} \underset{\mathscr{Q}}{\otimes} \overline{\mathcal{X}}, \mathcal{X} \stackrel{\otimes}{\mathscr{X}}) \equiv \mathcal{M}_{H}^{1}\left(H_{H}, H_{H}\right) \simeq H
$$

via the ring isomorphism defined by the left multiplication map $\Lambda: H \rightarrow A$ as in (3.41).

The base ring of the left bialgebroid underlying $\mathcal{A}$ is $L^{A}=\operatorname{BIM}\left(\mathcal{M}_{H}\right)^{2}(\mathcal{X}, \mathcal{X})$ that is

$$
L^{A}=\left\{\kappa \in \mathcal{M}_{H}^{1}\left(H_{H}, H_{H}\right) \mid \kappa\left(h_{1} * h_{2}\right)=\kappa\left(h_{1}\right) * h_{2} \quad \forall h_{1}, h_{2} \in H\right\} \simeq L
$$

via the ring isomorphism $\Lambda \circ s_{L}: L \rightarrow L^{A}$.

We claim that $\left(\Lambda: H \rightarrow A, \Lambda \circ s_{L}: L \rightarrow L^{A}\right)$ is a strict isomorphism of Hopf algebroids. Denote the convolution product (3.37) by $*$ and the one in (3.3) by $*_{A}$. Also write $S$ for the antipode in $\mathcal{H}$ and $S_{A}$ for the one in $\mathcal{A}$. Substituting the 2-morphisms (3.43), (4.45) (4.46) we obtain for all $h, k, m \in H$ the identities

$$
\begin{aligned}
S_{A} \circ \Lambda(h)(m) & =i^{(1)} s_{R} \circ \pi_{R}\left(\left(h i^{(2)}\right) * m\right)=\Lambda(S(h))(m) \\
{\left[\Lambda(h) *_{A} \Lambda(k)\right](m) } & =h m^{(1)} * k m^{(2)}=\Lambda(h * k)(m),
\end{aligned}
$$

where in the second step of the first line we used the left integral property of $i$. The identities (3.44) imply

$$
\gamma_{L}^{A} \circ \Lambda(h)=\Lambda(h) *_{A} S_{A}^{-1} \circ \Lambda\left(S\left(i_{(1)}\right)\right) \otimes \Lambda\left(i_{(2)}\right)=\Lambda\left(h * i_{(1)}\right) \otimes \Lambda\left(i_{(2)}\right)=(\Lambda \otimes \Lambda) \circ \gamma_{L}(h) .
$$

Computing the value of $\mu_{A}$ introduced in (3.9) on the element $\Lambda \circ s_{L}(l)$ of $L^{A}$, we have $\mu_{A}\left(\Lambda \circ s_{L}(l)\right)(h)=$ $t_{L}(l) h$ for all $h \in H$, hence

$$
\begin{aligned}
& s_{L}^{A} \circ \Lambda \circ s_{L}(l)=\Lambda \circ s_{L}(l) \\
& t_{L}^{A} \circ \Lambda \circ s_{L}(l)=\mu_{A} \circ \Lambda \circ s_{L}(l)=\Lambda \circ t_{L}(l) .
\end{aligned}
$$

Finally, for $h, k \in H$

$$
\left[\pi_{L}^{A} \circ \Lambda(h)\right](k)=h i * k=\left[\Lambda \circ s_{L} \circ \pi_{L}(h)\right](k) .
$$




\section{Appendix: The bicategory of internal bimodules}

Let $(\mathcal{M}, \otimes, U, \mathbf{l}, \mathbf{r}, \mathbf{a})$ be a monoidal category with coequalizers s.t. the monoidal product preserves the coequalizers. Then there is a bicategory $\operatorname{BIM}(\mathcal{M})$ of internal bimodules constructed as follows.

The 0-morphisms are the monoids in $\mathcal{M}$, the 1-morphisms with source $N$ and target $M$ the $M-N$ bimodules in $\mathcal{M}$ and the 2-morphisms the bimodule maps in $\mathcal{M}$. The vertical composition is given by the composition in $\mathcal{M}$. The horizontal composition is the tensor product of bimodules defined with the help of a coequalizer.

Let $\mathcal{R}=\left(R, m_{R}, \eta_{R}\right), \mathcal{S}=\left(S, m_{S}, \eta_{S}\right)$ and $\mathcal{T}=\left(T, m_{T}, \eta_{T}\right)$ be monoids, $\left(M, \lambda_{M}, \rho_{M}\right)$ an $\mathcal{R}-\mathcal{S}$ and $\left(N, \lambda_{N}, \rho_{N}\right)$ an $\mathcal{S}$ - $\mathcal{T}$-bimodule in $\mathcal{M}$. As an object in $\mathcal{M}$ the $M{ }_{S}^{\otimes} N$ be the object part of the coequalizer $\left(\tau_{M, N}, M \underset{S}{\otimes} N\right)$ :

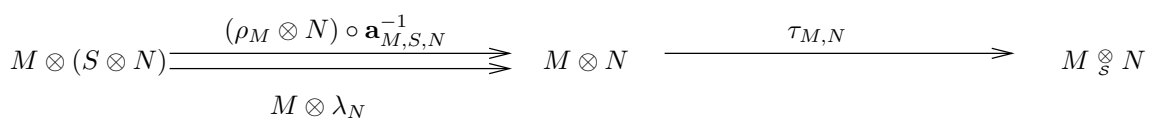

The object $M \underset{S}{\otimes} N$ can be equipped with an $\mathcal{R}-\mathcal{T}$ bimodule structure using the universal property of the coequalizer:

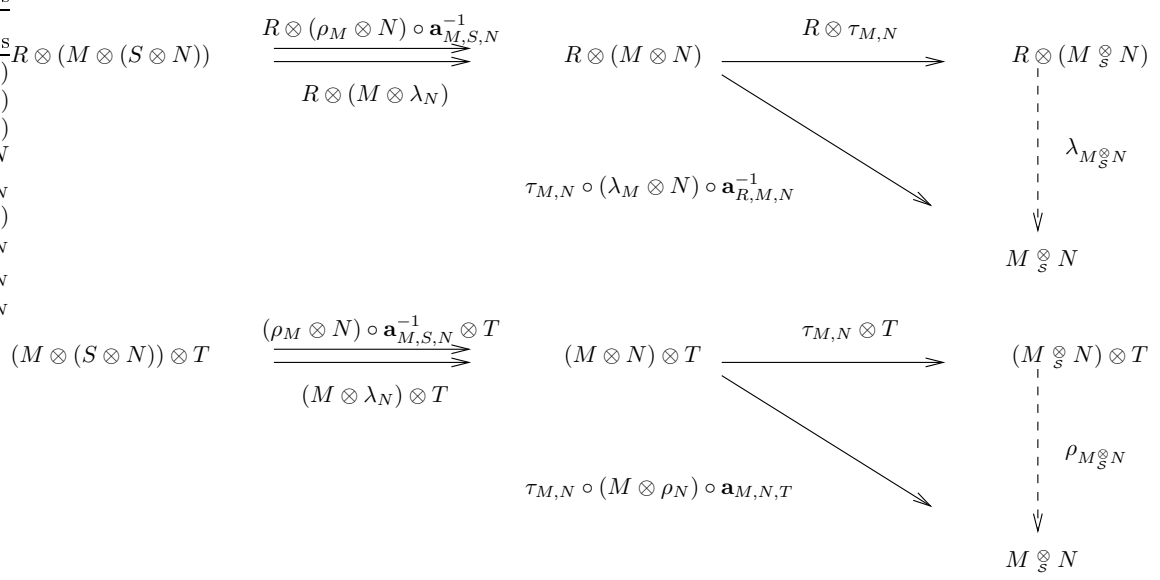

One checks that $\left(M \underset{\mathcal{S}}{\otimes} N, \lambda_{M_{\mathcal{S}}^{\otimes} N}, \rho_{M_{\mathcal{S}} N}\right)$ is an $\mathcal{R}-\mathcal{T}$ bimodule in $\mathcal{M}$.

For the 2-morphisms $p: M \rightarrow M^{\prime}$ and $q: N \rightarrow N^{\prime}$ the $\mathcal{S}$-module product $p \underset{\mathcal{S}}{\otimes} q$ is constructed also using the universality of the coequalizer:

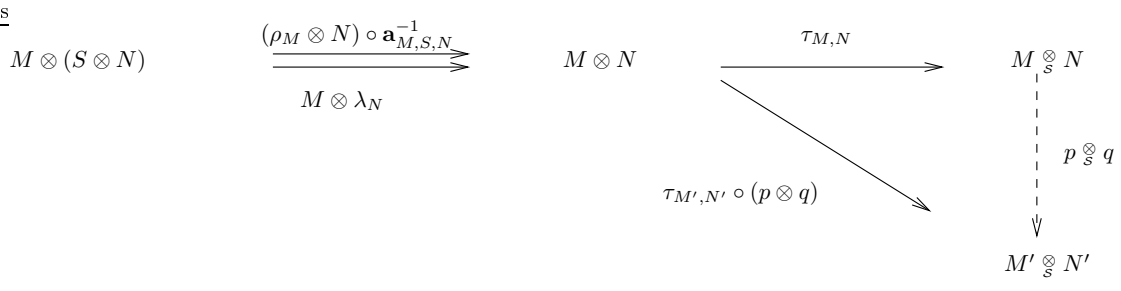

It is straightforward to check that $\mathbb{S}_{S}$ is functorial in both arguments and that the chosen coequalizer $\tau_{M, N}$ becomes a natural transformation from $\otimes$ to $\underset{S}{\otimes}$.

The coherence natural isomorphisms $\underline{\mathbf{a}}, \underline{\mathbf{l}}$ and $\underline{\mathbf{r}}$ are constructed also using the universality of the coequalizer: 


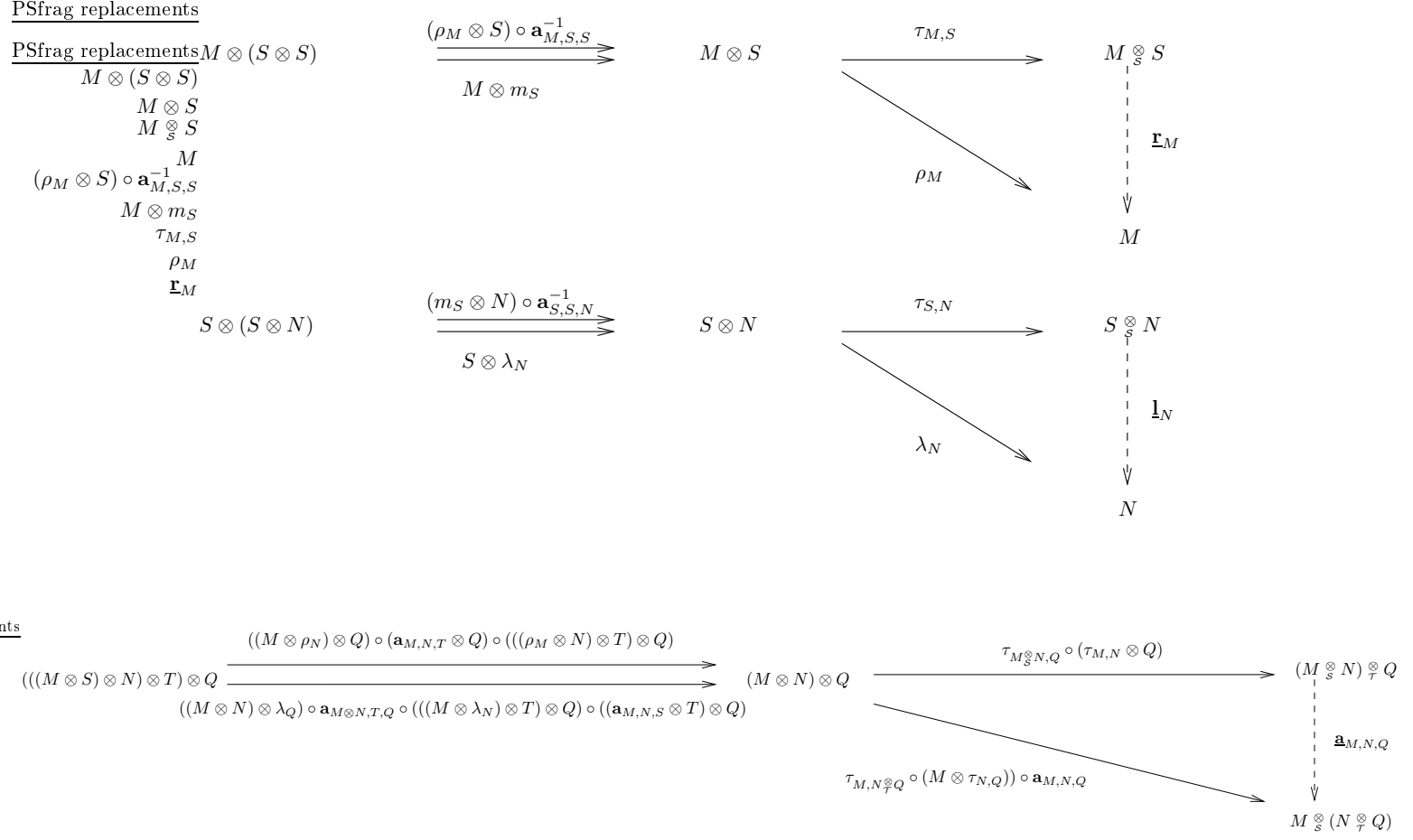

One checks that all $\underline{\mathbf{r}}_{M}, \underline{\mathbf{l}}_{N}$ and $\underline{\mathbf{a}}_{M, N, Q}$ are iso's, they are natural and satisfy the coherence axioms. This finishes the construction of the bicategory $\operatorname{BIM}(\mathcal{M})$. If the monoidal category $\mathcal{M}$ is additive then so is the bicategory $\operatorname{BIM}(\mathcal{M})$.

Proposition 4.1 Let $(\mathcal{M}, \otimes, U, \mathbf{l}, \mathbf{r}, \mathbf{a})$ be a monoidal category with coequalizers s.t. the monoidal product preserves the coequalizers. Then

i) $\mathcal{U}=\left(U, \mathbf{l}_{U}=\mathbf{r}_{U}, U\right)$ is a monoid in $\mathcal{M}$

ii) For any object $X$ of $\mathcal{M}$ the $\mathcal{U} X_{\mathcal{U}}=\left(X, \mathbf{l}_{X}, \mathbf{r}_{X}\right)$ is an $\mathcal{U}-\mathcal{U}$ bimodule in $\mathcal{M}$. Any morphism of $\mathcal{M}$ is an $\mathcal{U}-\mathcal{U}$ bimodule map. The tensor product over $\mathcal{U}$ in $\operatorname{BIM}(\mathcal{M})$ coincides with the monoidal product of $\mathcal{M}$.

iii) For a monoid $\mathcal{Q}=(Q, m, \eta)$ in $\mathcal{M}$ the

a) ${ }_{\mathcal{Q}} Q_{\mathcal{Q}}=(Q, m, m)$ is a $\mathcal{Q}-\mathcal{Q}$ bimodule in $\mathcal{M}$

b) $\mathcal{U} Q_{\mathcal{Q}}=\left(Q, \mathbf{l}_{Q}, m\right)$ is a $\mathcal{U}-\mathcal{Q}$ bimodule in $\mathcal{M}$

c) ${ }_{\mathcal{Q}} Q_{\mathcal{U}}=\left(Q, m, \mathbf{r}_{Q}\right)$ is a $\mathcal{Q}-\mathcal{U}$ bimodule in $\mathcal{M}$

d) The 1-morphism ${ }_{\mathcal{Q}} Q_{\mathcal{U}}$ is the left dual of $\mathcal{u} Q_{\mathcal{Q}}$ in the bicategory $\operatorname{BIM}(\mathcal{M})$

iv) For a Frobenius algebra $(\mathcal{Q}, \delta, \epsilon)$ in $\mathcal{M}$ the 1-morphism ${ }_{\mathcal{Q}} Q_{\mathcal{U}}$ is the two sided dual of $\mathcal{U} Q_{\mathcal{Q}}$ in the bicategory $\operatorname{BIM}(\mathcal{M})$.

The part iv) is a generalization of Yamagami's analogous result [31] where the same claim is proven under the additional assumption of split separability of the Frobenius algebra $(\mathcal{Q}, \delta, \epsilon)$.

Proof: The only non-trivial part of the statement is d) of iii) and $i v$ ). In order to prove $d$ ) of $i i i$ ) we construct the 2-morphisms

$$
\begin{aligned}
& \operatorname{BIM}(\mathcal{M})^{2}\left({ }_{\mathcal{Q}} Q_{\mathcal{U}} \stackrel{\otimes}{\mathcal{u}} \mathcal{u} Q_{\mathcal{Q}},{ }_{\mathcal{Q}} Q_{\mathcal{Q}}\right) \quad \ni \quad \operatorname{ev}_{L} \quad:=m \\
& \operatorname{BIM}(\mathcal{M})^{2}\left({ }_{\mathcal{U}} U_{\mathcal{U}}, \mathcal{U} Q_{\mathcal{Q}} \stackrel{\otimes}{\mathcal{Q}}_{\mathcal{Q}} Q_{\mathcal{U}}\right) \quad \ni \operatorname{coev}_{L}:=t^{-1} \circ \eta
\end{aligned}
$$

where $t$ is the 2-morphism $\underline{\mathbf{r}}_{\mathcal{Q} Q_{\mathcal{Q}}}=\underline{\mathbf{l}}_{\mathcal{Q}} Q_{\mathcal{Q}}$ regarded as a 2-morphism in $\operatorname{BIM}(\mathcal{M})\left(\mathcal{U}_{\mathcal{U}} Q_{\mathcal{Q}} \stackrel{\otimes}{\mathcal{Q}}_{\mathcal{Q}} Q_{\mathcal{U}}, \mathcal{U} Q_{\mathcal{U}}\right)$. The $\mathrm{ev}_{L}=m$ is a $\mathcal{Q}-\mathcal{Q}$ bimodule map by the associativity of $m$. 
By the unit property of $\eta$ the first two relations in (3.2) hold true.

Similarly, iv) follows by the existence of 2-morphisms

$$
\begin{aligned}
\operatorname{BIM}(\mathcal{M})^{2}\left({ }_{\mathcal{u}} Q_{\mathcal{Q}} \stackrel{\otimes}{\mathcal{Q}}_{\mathcal{Q}} Q_{\mathcal{U}}, \mathcal{U} U_{\mathcal{U}}\right) & \ni \quad \operatorname{ev}_{R}:=\varepsilon \circ t \\
\operatorname{BIM}(\mathcal{M})^{2}\left({ }_{\mathcal{Q}} Q_{\mathcal{Q}},{ }_{\mathcal{Q}} Q_{\mathcal{U}} \stackrel{\otimes}{\mathcal{U}} \mathcal{u} Q_{\mathcal{Q}}\right) & \ni \operatorname{coev}_{R}:=\delta .
\end{aligned}
$$

The $\operatorname{coev}_{R}=\delta$ is a $\mathcal{Q}-\mathcal{Q}$ bimodule map by the Frobenius algebra property. By the counit property of $\varepsilon$ the last two relations in (3.2) hold true.

\section{References}

[1] G. Böhm 'An alternative notion of Hopf algebroid' math.QA/0302325 to appear in the proceedings of "Hopf algebras in Noncommutative Geometry and Physics" 2002 Brussel, Marcel Dekker Lecture Notes series, ed.: S. Caenepeel and F. Van Oystaeyen

[2] G. Böhm, K. Szlachányi Hopf algebroids with bijective antipodes: axioms, integrals and duals, math.QA/0302325

[3] G. Böhm, F. Nill, K. Szlachányi 'Weak Hopf Algebras I: Integral Theory and $C^{*}$-structure' J. Algebra 221 (1999) p. 385

[4] G. Böhm, K. Szlachányi 'A Coassociative $C^{*}$-Quantum Group with Non-Integral Dimensions' Lett. Math. Phys. 35 (1996) p. 137

[5] T. Brezinski, G. Militaru 'Bialgebroids, $\times_{R}$-bialgebras and Duality' J. Algebra 247 No.2 (2002) p. 467

[6] A. Connes, H. Moscovici 'Cyclic cohomology and Hopf algebra symmetry' Conference Mosh Flato 1999, Dijon Lett. Math. Phys. 52 No.1 (2000) p.1

[7] M-C. David 'Paragroupe d' Adrian Ocneanu et algebre de Kac' Pacif. J. of Math. 172 No. 2 (1996) p.331

[8] M. Enock, R. Nest 'Inclusions of Factors, Multiplicative Unitaries and Kac Algebras' J. Funct. Analysis 137 (1996) p.466

[9] M. Enock, J-M. Vallin 'Inclusions of von Neumann algebras and quantum groupoids' Inst. de Math. de Jussieu preprint No. 156 (1998) and M. Enock 'Inclusions of von Neumann algebras and quantum groupoids II.' Inst. de Math. de Jussieu preprint No. 231 (1999)

[10] R. Herrmann, A. Ocneanu 'Index Theory and Galois Theory for Infinite Inclusions of Factors' C.R. Acad. Sci. Paris 309 (1989) p.923

[11] L. Kadison, K. Szlachányi 'Dual Bialgebroids for Depth Two Ring Extensions' math.RA/0108067 to appear in Advances in Mathematics

[12] R. Longo 'A duality for Hopf algebras and for subfactors I.' Commun. Math. Phys. 159 (1994) p.133

[13] J. H. Lu 'Hopf Algebroids and Quantum Groupoids' Int. J. Math. Vol. 7 No. 1 (1996) p. 47

[14] M. Müger 'From Subfactors to Categories and Topology I: Frobenius Algebras in and Morita Equivalence of Tensor Categories' J. Pure Appl. Alg. 180 (2003) p. 81

[15] D. Nikshych, L. Vainerman 'A characterization of depth 2 subfactors of $I I_{1}$ factors ' J. Func. Analysis, 171 no.2 (2000) p.278

[16] D. Nikshych, L. Vainerman 'Galois correspondence for $I I_{1}$ factors and quantum groupoids' Journal of Func. Analysis, 178 no.1 (2000) p.113 
[17] F. Nill 'Weak Bialgebras' math.QA/9805104

[18] F. Nill, K. Szlachányi, H-W. Wiesbrock 'Weak Hopf Algebras and Reducible Jones Inclusions of Depth 2. I. math.QA/9806130 and F. Nill, K. Szlachányi, H-W. Wiesbrock 'Weak Hopf Algebras and Reducible Jones Inclusions of Depth 2. II. unpublished

[19] F. Nill, H-W Wiesbrock 'A comment on Jones Inclusions with finite Index' Rev. Math. Phys. 7 (1995) p.599

[20] A. Ocneanu 'A Galois Theory for Operator Algebras' Notes of a Lecture. Quantum Symmetry, Differential Geometry of Finite Graphs and Classification Subfactors, Lectures by A. Ocneanu given at the University of Tokyo, Notes taken by Y. Kawahigashi (1992)

[21] P. Schauenburg 'Bialgebras over noncommutative rings, and a structure theorem for Hopf bimodules' Applied Categorical Structures 6 (1998) p.193

[22] P. Schauenburg 'Duals and Doubles of Quantum Groupoids' in: "New trends in Hopf algebra theory" Proceedings of the Colloquium on quantum groups and Hopf algebras, La Falda, Sierra de Cordoba, Argentina, 1999, AMS Contemporary Mathematics 267 (2000) p. 273

[23] P. Schauenburg 'Weak Hopf Algebras and Quantum Groupoids' math.QA/0204180

[24] M. E. Sweedler 'Groups of simple algebras' Publ. Math. I.H.E.S. 44 (1974) p.79

[25] K. Szlachányi: 'Finite Quantum Groupoids and Inclusions of Finite Type' Fields Institute Communications Vol 30 (2001) p.393

[26] K. Szlachányi 'Galois actions by finite quantum groupoids' in "Locally Compact Quantum Groups and Groupoids", proceedings of the meeting of theoretical physicists and mathematicians, Strasbourg, February 2002, IRMA Lectures in Mathematics and Theoretical Physics 2, ed.: L. Vainerman, series editor: V. Turaev, de Gruyter 2003

[27] W. Szymański 'Finite Index Subfactors and Hopf Algebra Crossed Products'Proc. Amer. Math. Soc. 120 No. 2 (1994) p. 519

[28] M. Takeuchi 'Groups of Algebras over $A \otimes \bar{A}$ ' J. Math. Soc Japan 29 (1997) p. 459

[29] P. Xu 'Quantum Groupoids' Commun. Math. Phys. 216 (2001) p. 539

[30] S. Yamagami 'On Ocneanu's Characterization of Crossed Products' preprint (1993)

[31] S. Yamagami 'Frobenius algebras in tensor categories' unpublished

[32] D. N. Yetter 'Framed tangles and a theorem of Deligne on braided deformations of tannakian categories' Contemp. Math. 134 (1992) p. 325

[33] Y. Watatani 'Index for $C^{*}$-subalgebras' Memoirs of the AMS Vol. 23 No. 424 (1990) 